\title{
Point processes in arbitrary dimension from fermionic gases, random matrix theory, and number theory
}

\author{
Salvatore Torquato ${ }^{1}$, A Scardicchio $^{2}$ and Chase E Zachary ${ }^{3}$ \\ ${ }^{1}$ Department of Chemistry, Princeton University, Princeton, New Jersey 08544, USA; \\ Program in Applied and Computational Mathematics, Princeton University, \\ Princeton, New Jersey 08544, USA; \\ Princeton Institute for the Science and Technology of Materials, Princeton \\ University, Princeton, New Jersey 08544, USA; \\ Princeton Center for Theoretical Science, Princeton University, Princeton, New \\ Jersey 08544, USA; and School of Natural Sciences, Institute for Advanced Study, \\ Princeton, New Jersey, 08540, USA \\ E-mail: torquato@princeton.edu \\ 2 Department of Physics, Joseph Henry Laboratories, Princeton University, \\ Princeton, New Jersey 08544, USA; and Princeton Center for Theoretical Science, \\ Princeton University, Princeton, New Jersey 08544, USA \\ E-mail: ascardic@princeton.edu \\ ${ }^{3}$ Department of Chemistry, Princeton University, Princeton, New Jersey 08544, USA \\ E-mail: czachary@princeton.edu
}

\begin{abstract}
.
It is well known that one can map certain properties of random matrices, fermionic gases, and zeros of the Riemann zeta function to a unique point process on the real line $\mathbb{R}$. Here we analytically provide exact generalizations of such a point process in $d$-dimensional Euclidean space $\mathbb{R}^{d}$ for any $d$, which are special cases of determinantal processes. In particular, we obtain the $n$-particle correlation functions for any $n$, which completely specify the point processes in $\mathbb{R}^{d}$. We also demonstrate that spinpolarized fermionic systems in $\mathbb{R}^{d}$ have these same $n$-particle correlation functions in each dimension. The point processes for any $d$ are shown to be hyperuniform, i.e., infinite wavelength density fluctuations vanish, and the structure factor (or power spectrum) $S(k)$ has a nonanalytic behavior at the origin given by $S(k) \sim|k|(k \rightarrow 0)$. The latter result implies that the pair correlation function $g_{2}(r)$ tends to unity for large pair distances with a decay rate that is controlled by the power law $1 / r^{d+1}$, which is a well-known property of bosonic ground states and more recently has been shown to characterize maximally random jammed sphere packings. We graphically display one- and two-dimensional realizations of the point processes in order to vividly reveal their "repulsive" nature. Indeed, we show that the point processes can be characterized by an effective "hard-core" diameter that grows like the square root of $d$. The nearest-neighbor distribution functions for these point processes are also evaluated and rigorously bounded. Among other results, this analysis reveals that the probability of finding a large spherical cavity of radius $r$ in dimension $d$ behaves like a Poisson point process but in dimension $d+1$, i.e., this probability is given by $\exp \left[-\kappa(d) r^{d+1}\right]$
\end{abstract}


for large $r$ and finite $d$, where $\kappa(d)$ is a positive $d$-dependent constant. We also show that as $d$ increases, the point process behaves effectively like a sphere packing with a coverage fraction of space that is no denser than $1 / 2^{d}$. This coverage fraction has a special significance in the study of sphere packings in high-dimensional Euclidean spaces.

PACS numbers: 02.50.Ey, 05.40.-a, 05.30.Fk

Submitted to: Journal of Statistical Mechanics: Theory and Experiment

Keywords: Correlation functions, Point processes, Fermions, Random matrix theory, Number theory 


\section{Introduction}

It is well known that there is a remarkable connection between the statistical properties of certain random Hermitian matrices, the zeros of the Riemann zeta function, and fermionic gases [1, 3, 2, 4, 5, 6]. Underlying each of these objects are certain onedimensional point processes (defined more precisely in Section 2) whose statistical properties (under certain limits) are believed to be identical. The purpose of this paper is to provide generalizations of this unique point process to point processes in $d$-dimensional Euclidean space $\mathbb{R}^{d}$ for any $d$, and to characterize their spatial statistics exactly. Since the characterization of a point process can be viewed as the study of a system of interacting "point" particles, exact descriptions of nontrivial point processes in arbitrary space dimension, which are hard to come by [8], are of great value in the field of statistical mechanics.

There are three prominent theories of random Hermitian matrices, which model the Hamiltonians of certain random dynamical systems; see the excellent book by Mehta [3]. If the dynamical system is symmetric under time reversal, then the relevant theory for integral spin is that of the Gaussian orthogonal ensemble (GOE) or the Gaussian symplectic ensemble (GSE) for half-integer spin. On the other hand, the Gaussian unitary ensemble (GUE) models random Hamiltonians without time reversal symmetry, which is relevant to certain properties of the Riemann zeta function. Although there are distinct one-dimensional point processes associated with each of these ensembles, our interest here will be in the GUE because of its relationship to the Riemann zeta function.

The GUE of degree $N$ consists of the set of all $N \times N$ Hermitian matrices together with a Haar measure. This is the unique probability measure on the set of $N \times N$ Hermitian matrices that is invariant under conjugation by unitary matrices such that the individual matrix entries are independent random variables. Dyson [1] showed that the eigenvalue distrubutions of the GUE are closely related to those of the "circular unitary ensemble" (CUE), which he exactly mapped into a problem of point particles on a unit circle interacting with a two-dimensional Coulombic force law at a particular temperature. This point process on the unit circle or, equivalently, on the real line $\mathbb{R}$ in the large- $N$ limit (when suitably normalized) has a pair correlation function (defined in Section 2 ) in $\mathbb{R}$ at number density $\rho=1$ given by

$$
g_{2}(r)=1-\frac{\sin ^{2}(\pi r)}{(\pi r)^{2}}
$$

Equation (1) also applies for the GUE in the limit $N \rightarrow+\infty$ such that the mean gap distance between eigenvalues at the origin is normalized; this limit has the effect of magnifying the bulk of the eigenvalue density on $\mathbb{R}$ such that (1) is well-defined. We see that this point process is always negatively correlated, i.e., $g_{2}(r)$ never exceeds unity (see Fig. 1) and is "repulsive" in the sense that $g_{2}(r) \rightarrow 0$ as $r$ tends to zero. More generally, Dyson [2] proved that the $n$-particle correlation function (defined in Section 
2) is given by the following determinant:

$$
g_{n}\left(r_{12}, r_{13}, \ldots, r_{1 n}\right)=\operatorname{det}\left(\frac{\sin \left(\pi r_{i j}\right)}{\pi r_{i j}}\right)_{i, j=1, \ldots, n} .
$$
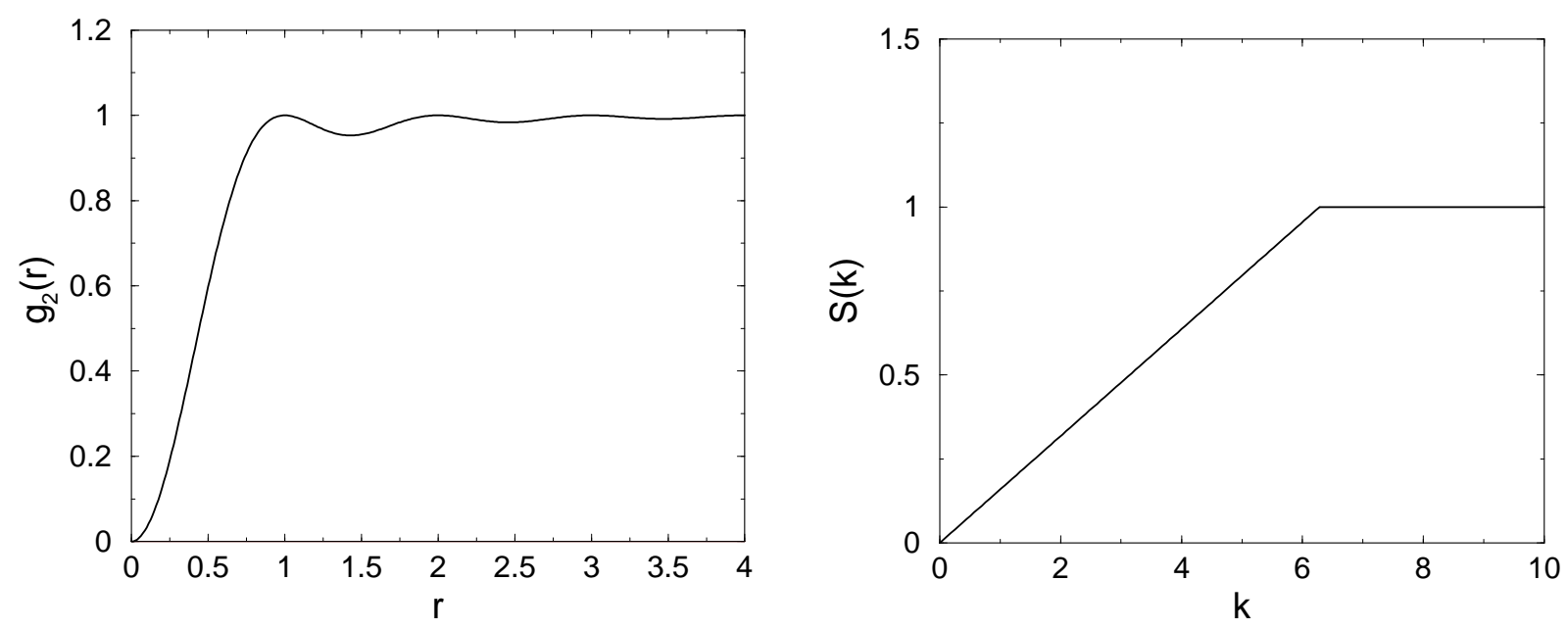

Figure 1. Left panel: The pair correlation function $g_{2}(r)$ versus distance $r$ for the eigenvalues of the GUE/CUE in the large- $N$ limit, which is conjectured to be the same as the one characterizing the nontrivial zeros of the Riemann zeta function. Spinpolarized fermions in their ground state in $\mathbb{R}$ have the same pair correlation function. Right panel: The corresponding spectral counterpart, called the structure factor $S(k)$ [cf. (7)], as a function of wavenumber $k$.

Montgomery [4] conjectured that the pair correlation function of nontrivial zeros of the Riemann zeta function (which, according to the Riemann hypothesis, lie along the critical line $1 / 2+i t$ with $t \in \mathbb{R}$ ) is given exactly by the GUE/CUE function (1) in the asymptotic limit (high on the critical line) when appropriately normalized. This remarkable correspondence was further established by Odlyzko [5], who numerically verified the Riemann hypothesis for the first $10^{13}$ nontrivial zeros of the zeta function as well as at much larger heights and confirmed that the pair correlation function agrees with (1). Rudnick and Sarnak [6] proved that, under the Riemann hypothesis, the nontrivial zeros have $n$-particle densities for any $n$ given by (2). The reader is referred to the excellent review article by Katz and Sarnak [7], which discusses the connection between the zeros of zeta functions and classical symmetric groups, of which the three canonical random-matrix ensembles are but special cases.

For spin-polarized free fermions in $\mathbb{R}$ (fermion gas) at number density $\rho=1$, it is known that the pair correlation function in the ground state (i.e., completely filling the Fermi "sphere") is given by

$$
g_{2}(r)=1-\frac{\sin ^{2}\left(k_{F} r\right)}{\left(k_{F} r\right)^{2}},
$$

where $k_{F}$ is the Fermi radius, which is the one-dimensional analog of the well-known three-dimensional result [9]. Therefore, we see that when $k_{F}=\pi$, we obtain the 
GUE/CUE pair correlation function (11). The repulsive nature of the point process in this context arises physically from the Pauli exclusion principle.

Very little is known about how to generalize one-dimensional point processes associated with random matrices and number-theoretic functions to higher dimensions. In this paper, we analytically obtain exact generalizations of the aforementioned onedimensional point process in $d$-dimensional Euclidean space $\mathbb{R}^{d}$ for any $d$. These processes are special cases of determinantal processes. In particular, we obtain the $n$-particle correlation functions for any $n$, which completely specify the point processes in $\mathbb{R}^{d}$. We also show that spin-polarized fermionic systems in $\mathbb{R}^{d}$ have these same $n$ particle correlation functions in each dimension. We show that the point processes for any $d$ are hyperuniform, i.e., infinite wavelength density fluctuations vanish, and the structure factor (or power spectrum) $S(k)$ has a nonanalytic behavior at the origin given by $S(k) \sim|k|(k \rightarrow 0)$. The latter result implies that the pair correlation function $g_{2}(r)$ tends to unity for large pair distances with a decay rate that is controlled by the power law $1 / r^{d+1}$. In three dimensions, such a dominant power-law decay of $g_{2}(r)$ is a well-known property of bosonic systems in their ground states [10, 12] and, more recently, has been shown to characterize maximally random jammed sphere packings [13].

Realizations of the point processes are displayed in one and two dimensions, using a simulation technique described by us elsewhere [14, which vividly reveal their "repulsive" nature. In fact, we show the point processes can be characterized by an effective "hard-core" diameter that grows like the square root of $d$. The nearestneighbor distribution functions for these point processes are also studied by evaluating and rigorously bounding them. Among other results, this analysis reveals that the probability of finding a large spherical cavity of radius $r$ in dimension $d$ behaves like a Poisson point process but in dimension $d+1$, i.e., this probability is given by $\exp \left[-\kappa(d) r^{d+1}\right]$ for large $r$, where $\kappa(d)$ is a positive $d$-dependent constant. We also show that as $d$ increases, the point process behaves effectively like a sphere packing with a coverage fraction of space that is no denser than $1 / 2^{d}$. As we will see, this value of the coverage fraction has a special significance in the study of sphere packings in high dimensions.

In Section 2, we present background and definitions concerning point processes that are particularly germane to the ensuing analysis. Section 3 discusses general determinantal point processes. In Section 4, we obtain the determinantal point processes in $\mathbb{R}^{d}$ that are generalizations of the aforementioned one-dimensional point process associated with fermions, random matrices, and the Riemann zeta function. We call the most general of these point processes "Fermi-shells" point processes. The asymptotic properties of various pair statistics are investigated. We show that spin-polarized fermionic systems in $\mathbb{R}^{d}$ have the same $n$-particle correlation functions in each dimension. Section 5 analyzes various nearest-neighbor functions for the special case of the "Fermisphere" point processes in $\mathbb{R}^{d}$. We present concluding comments in Section 6, including remarks about possible connections of our point processes to random matrix and number 
theory.

\section{Background on Point Processes}

\subsection{Definitions}

A stochastic point process in $\mathbb{R}^{d}$ is defined as a mapping from a probability space

to configurations of points $\mathbf{x}_{1}, \mathbf{x}_{2}, \mathbf{x}_{3} \ldots$ in $d$-dimensional Euclidean space $\mathbb{R}^{d}$. More precisely, let $X$ denote the set of configurations such that each configuration $x \in X$ is a subset of $\mathbb{R}^{d}$ that satisfies two regularity conditions: (i) there are no multiple points $\left(\mathbf{x}_{i} \neq \mathbf{x}_{j}\right.$ if $\left.i \neq j\right)$ and (ii) each bounded subset of $\mathbb{R}^{d}$ must contain only a finite number of points of $x$. We denote by $N(B)$ the number of points within $x \cap B, B \in \mathcal{B}$, where $\mathcal{B}$ is the ring of bounded Borel sets in $\mathbb{R}^{d}$. Thus, we always have $N(B)<\infty$ for $B \in \mathcal{B}$, but the possibility $N\left(\mathbb{R}^{d}\right)=\infty$ is not excluded. We note that there exists a minimal $\sigma$-algebra $\mathcal{U}$ of subsets of $X$ that renders all of the functions $N(B)$ measurable. Let $(\Omega, \mathcal{F}, \mathcal{P})$ be a probability space. Any measurable map $x: \Omega \rightarrow X$ is called a stochastic point process [15, 16]. Henceforth, we will simply call this map a point process. Note that this random setting is quite general. It incorporates cases in which the location of the points are deterministically known, such as a lattice.

A point process is completely statistically characterized by specifying the countably infinite set of $n$-particle probability density functions $\rho_{n}\left(\mathbf{r}_{1}, \mathbf{r}_{2}, \ldots, \mathbf{r}_{n}\right)(n=$ $1,2,3 \ldots)$ [16]. The distribution-valued function $\rho_{n}\left(\mathbf{r}_{1}, \mathbf{r}_{2}, \ldots, \mathbf{r}_{n}\right)$ has a probabilistic interpretation: apart from trivial constants, it is the probability density function associated with finding $n$ different points at positions $\mathbf{r}_{1}, \ldots, \mathbf{r}_{n}$ and hence has the nonnegativity property

$$
\rho_{n}\left(\mathbf{r}_{1}, \mathbf{r}_{2}, \ldots, \mathbf{r}_{n}\right) \geq 0 \quad \forall \mathbf{r}_{i} \in \mathbb{R}^{d} \quad(i=1,2, \ldots n) .
$$

The point process is statistically homogeneous or translationally invariant if for every constant vector $\mathbf{y}$ in $\mathbb{R}^{d}, \rho_{n}\left(\mathbf{r}_{1}, \mathbf{r}_{2}, \ldots, \mathbf{r}_{n}\right)=\rho_{n}\left(\mathbf{r}_{1}+\mathbf{y}, \ldots, \mathbf{r}_{n}+\mathbf{y}\right)$, which implies that

$$
\rho_{n}\left(\mathbf{r}_{1}, \mathbf{r}_{2}, \ldots, \mathbf{r}_{n}\right)=\rho^{n} g_{n}\left(\mathbf{r}_{12}, \ldots, \mathbf{r}_{1 n}\right),
$$

where $\rho$ is the number density (number of points per unit volume in the infinite-volume limit) and $g_{n}\left(\mathbf{r}_{12}, \ldots, \mathbf{r}_{1 n}\right)$ is the $n$-particle correlation function, which depends on the relative positions $\mathbf{r}_{12}, \mathbf{r}_{13}, \ldots$, where $\mathbf{r}_{i j} \equiv \mathbf{r}_{j}-\mathbf{r}_{i}$ and we have chosen the origin to be at $\mathbf{r}_{1}$. We call $g_{2}(\mathbf{r})=g_{2}(-\mathbf{r})$ the pair correlation function.

For translationally invariant point processes without long-range order, $g_{n}\left(\mathbf{r}_{12}, \ldots, \mathbf{r}_{1 n}\right) \rightarrow$ 1 when the points (or "particles") are mutually far from one another, i.e., as $\left|\mathbf{r}_{i j}\right| \rightarrow \infty$ $(1 \leq i<j<\infty), \rho_{n}\left(\mathbf{r}_{1}, \mathbf{r}_{2}, \ldots, \mathbf{r}_{n}\right) \rightarrow \rho^{n}$. Thus, the deviation of $g_{n}$ from unity provides a measure of the degree of spatial correlation between the particles with unity corresponding to no spatial correlation. Note that for a translationally invariant Poisson point process, $g_{n}$ is unity for all values of its argument.

It is useful to introduce the total correlation function $h(\mathbf{r})$ of a translationally invariant point process, which is related to the pair correlation function via

$$
h(\mathbf{r}) \equiv g_{2}(\mathbf{r})-1
$$


and decays to zero for large $|\mathbf{r}|$ in the absence of long-range order. Note that $h(\mathbf{r})=0$ for all $\mathbf{r}$ for a translationally invariant Poisson point process. An important nonnegative spectral function $S(\mathbf{k})$, called the structure factor (or power spectrum), is defined as follows:

$$
S(\mathbf{k})=1+\rho \tilde{h}(\mathbf{k})
$$

where $\tilde{h}(\mathbf{k})$ is the Fourier transform of $h(\mathbf{r})$. For a translationally and rotationally invariant point process, it is useful to consider the cumulative coordination number $Z(r)$, defined to be the expected number of points found in a sphere of radius $r$ centered at an arbitrary point of the point process, which is related to the pair correlation function as follows:

$$
Z(r)=\rho s_{1}(1) \int_{0}^{r} x^{d-1} g_{2}(x) \mathrm{d} x
$$

where

$$
s_{1}(r)=\frac{2 \pi^{d / 2} r^{d-1}}{\Gamma(d / 2)}
$$

is the surface area of a $d$-dimensional sphere of radius $r$. It is clear that since $g_{2}(r)$ is a nonnegative function, $Z(r)$ is a monotonically increasing function of $r$.

The Fourier transform of an $L^{1}$ function $f: \mathbb{R}^{d} \rightarrow \mathbb{R}$ is defined by

$$
\tilde{f}(\mathbf{k})=\int_{\mathbb{R}^{d}} f(\mathbf{r}) \exp [-\mathrm{i}(\mathbf{k} \cdot \mathbf{r})] \mathrm{d} \mathbf{r},
$$

where $L^{1}$ denotes the space of absolutely integrable functions on $\mathbb{R}^{d}$. If $f: \mathbb{R}^{d} \rightarrow \mathbb{R}$ is a radial function, i.e., $f$ depends only on the modulus $r=|\mathbf{r}|$ of the vector $\mathbf{r}$, then its Fourier transform is given by

$$
\tilde{f}(k)=(2 \pi)^{\frac{d}{2}} \int_{0}^{\infty} r^{d-1} f(r) \frac{J_{(d / 2)-1}(k r)}{(k r)^{(d / 2)-1}} \mathrm{~d} r,
$$

where $k$ is the modulus of the wave vector $\mathbf{k}$ and $J_{\nu}(x)$ is the Bessel function of order $\nu$. The inverse transform of $\tilde{f}(k)$ is given by

$$
f(r)=\frac{1}{(2 \pi)^{\frac{d}{2}}} \int_{0}^{\infty} k^{d-1} \tilde{f}(k) \frac{J_{(d / 2)-1}(k r)}{(k r)^{(d / 2)-1}} \mathrm{~d} k .
$$

\subsection{Number Variance and Hyperuniformity}

We denote by $\sigma^{2}(A)$ the variance in the number of points $N(A)$ contained within a window $A \subset \mathbb{R}^{d}$. The number variance $\sigma^{2}(A)$ for a specific choice of $A$ is necessarily a positive number and is generally related to the total correlation function $h(\mathbf{r})$ for a translationally invariant point process [18]. In the special case of a spherical window of radius $R$ in $\mathbb{R}^{d}$, it is explicitly given by

$$
\sigma^{2}(R)=\rho v_{1}(R)\left[1+\rho \int_{\mathbb{R}^{d}} h(\mathbf{r}) \alpha(r ; R) \mathrm{d} \mathbf{r}\right],
$$

where $\sigma^{2}(R)$ is the number variance for a spherical window of radius $R$ in $\mathbb{R}^{d}$ and $\alpha(r ; R)$ is the volume common to two spherical windows of radius $R$ whose centers are separated 
by a distance $r$ divided by $v_{1}(R)$, the volume of a spherical window of radius $R$, given explicitly by

$$
v_{1}(R)=\frac{\pi^{d / 2}}{\Gamma(1+d / 2)} R^{d}
$$

We will call $\alpha(r ; R)$ the scaled intersection volume.

For large $R$, it has been proved that $\sigma^{2}(R)$ cannot grow more slowly than $\gamma R^{d-1}$, where $\gamma$ is a positive constant [17]. We note that point processes (translationally invariant or not) for which $\sigma^{2}(R)$ grows more slowly than the window volume (i.e., as $R^{d}$ ) for large $R$ are examples of hyperuniform (or superhomogeneous) point patterns [18, 19]. This classification includes all periodic point processes [18], certain aperiodic point processes [19, 18], one-component plasmas [19, 18], point processes associated with a wide class of tilings of space [20, 21], and certain disordered sphere packings [16, 22, 23, 24]. Hyperuniformity implies that the structure factor $S(\mathbf{k})$ has the following small k behavior:

$$
\lim _{\mathbf{k} \rightarrow \mathbf{0}} S(\mathbf{k})=0 .
$$

The scaled intersection volume $\alpha(r ; R)$ appearing in (13) and its associated Fourier transform will play a prominent role in this paper. The former quantity is defined by a convolution integral involving the indicator function $w$ for a $d$-dimensional spherical "window" of radius $R$ centered at position $\mathbf{x}_{0}[18$, i.e.,

$$
w\left(\left|\mathbf{x}-\mathbf{x}_{0}\right| ; R\right)=\Theta\left(R-\left|\mathbf{x}-\mathbf{x}_{0}\right|\right),
$$

where $\Theta(x)$ is the Heaviside step function

$$
\Theta(x)= \begin{cases}0, & x<0 \\ 1, & x \geq 0\end{cases}
$$

Specifically, the scaled intersection volume is given by

$$
\alpha(r ; R)=\frac{1}{v_{1}(R)} \int_{\mathbb{R}^{d}} w\left(\mathbf{r}_{1}-\mathbf{x}_{0} ; \mathbf{R}\right) w\left(\mathbf{r}_{2}-\mathbf{x}_{0} ; \mathbf{R}\right) \mathrm{d} \mathbf{x}_{0} .
$$

The scaled intersection volume has the support $[0,2 R]$, the range $[0,1]$, and the following alternative integral representation [16]:

$$
\alpha(r ; R)=c(d) \int_{0}^{\cos ^{-1}[r /(2 R)]} \sin ^{d}(\theta) \mathrm{d} \theta,
$$

where $c(d)$ is the $d$-dimensional constant given by

$$
c(d)=\frac{2 \Gamma(1+d / 2)}{\pi^{1 / 2} \Gamma[(d+1) / 2]} .
$$

Torquato and Stillinger [16] found the following series representation of the scaled intersection volume $\alpha(r ; R)$ for $r \leq 2 R$ and for any $d$ :

$$
\alpha(r ; R)=1-c(d) x+c(d) \sum_{n=2}^{\infty}(-1)^{n} \frac{(d-1)(d-3) \cdots(d-2 n+3)}{(2 n-1)[2 \cdot 4 \cdot 6 \cdots(2 n-2)]} x^{2 n-1}
$$


where $x=r /(2 R)$. For even dimensions, relation (21) is an infinite series, but for odd dimensions, the series truncates such that $\alpha(r ; R)$ is a univariate polynomial of degree $d$. In even dimensions, the scaled intersection volume involves transcendental functions (e.g., for $d=2, \alpha(r ; R)=2 \pi^{-1}\left[\cos ^{-1}\left(\frac{r}{2 R}\right)-\frac{r}{2 R}\left(1-\frac{r^{2}}{4 R^{2}}\right)^{1 / 2}\right]$ for $0 \leq r \leq 2 R$ ). Figure 2 provides plots of $\alpha(r ; R)$ as a function of $r$ for the first five space dimensions. For any dimension, $\alpha(r ; R)$ is a monotonically decreasing function of $r$. At a fixed value of $r$ in the interval $(0,2 R), \alpha(r ; R)$ is a monotonically decreasing function of the dimension $d$.

The Fourier transform of the scaled intersection volume function (19), which is given by

$$
\tilde{\alpha}(k ; R)=\frac{[\tilde{w}(k ; R)]^{2}}{v_{1}(R)},
$$

where

$$
\begin{aligned}
\tilde{w}(k ; R) & =\frac{(2 \pi)^{d / 2}}{k^{(d / 2)-1}} \int_{0}^{R} r^{d / 2} J_{(d / 2)-1}(k r) \mathrm{d} r \\
& =\left(\frac{2 \pi}{k R}\right)^{d / 2} R^{d} J_{d / 2}(k R),
\end{aligned}
$$

is the Fourier transform of the window indicator function (16). Therefore, the Fourier transform of $\alpha(r ; R)$ is the following nonnegative function of $k$ :

$$
\tilde{\alpha}(k ; R)=2^{d} \pi^{d / 2} \Gamma(1+d / 2)\left(\frac{J_{d / 2}(k R)}{k^{d / 2}}\right)^{2} .
$$
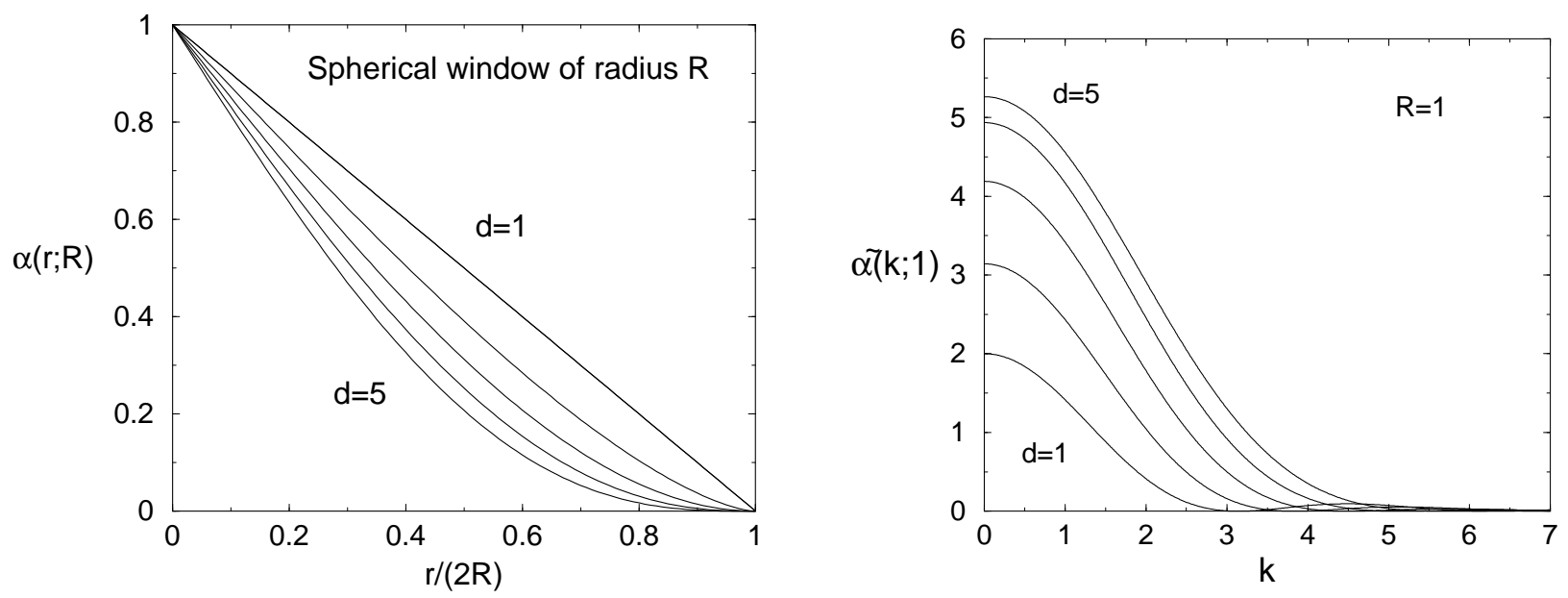

Figure 2. Left panel: The scaled intersection volume $\alpha(r ; R)$ for spherical windows of radius $R$ as a function of $r$ for the first five space dimensions. The uppermost curve is for $d=1$ and lowermost curve is for $d=5$. Right panel: Corresponding Fourier transforms for the case $R=1$. The uppermost curve is for $d=5$ and lowermost curve is for $d=1$.

It has been shown that finding the point process that minimizes the number variance $\sigma^{2}(R)$ is equivalent to finding the ground state of a certain repulsive pair potential with compact support [18]. This problem is directly related to an outstanding problem in 
number theory involving generalized zeta functions and lattices [25]. Understanding such ground states can be facilitated by utilizing "duality" relations that link ground states in real space to those in Fourier space [26].

\section{Determinantal Point Processes}

We will be able to obtain the appropriate $d$-dimensional generalizations of point processes corresponding to the eigenvalues of the GUE, the zeros of the Riemann zeta function, or the one-dimensional fermionic gas by appealing to the notion of a determinantal point process in $\mathbb{R}^{d}$ [27]. Determinantal point processes were introduced by Macchi [27, who originally called them fermion point processes. Soshnikov [28] presented a review of this subject and discussed applications to random matrix theory, statistical mechanics, quantum mechanics, and representation theory. It is also noteworthy that examples of determinantal point processes arise in self-avoiding random walks [30] and uniform spanning trees [31].

Without loss of generality, the number density is set to unity $(\rho=1)$ in the ensuing discussion. Let $H(\mathbf{r})=H(-\mathbf{r})$ be a translationally invariant Hermitian-symmetric kernel of an integral operator $\mathcal{H}$. A translationally invariant determinantal point process in $\mathbb{R}^{d}$ exists if the the $n$-particle density functions for $n \geq 1$ are given by the following determinants:

$$
\rho_{n}\left(\mathbf{r}_{12}, \mathbf{r}_{13}, \ldots, \mathbf{r}_{1 n}\right)=\operatorname{det}\left[H\left(\mathbf{r}_{i j}\right)\right]_{i, j=1, \ldots, n},
$$

where $H(\mathbf{0})=1$. By virtue of the nonnegativity of the $\rho_{n}$ in the pointwise sense [cf. (4)] and (25), it follows that $\mathcal{H}$ must have nonnegative minors and $\mathcal{H}$ must be a nonnegative operator, which implies that $H(\mathbf{r})$ is positive semidefinite. The latter implies that the Fourier transform $\tilde{H}(\mathbf{k})$ of the kernel $H(\mathbf{r})$ is nonnegative, and this property together with the condition $H(\mathbf{0})=1=\int_{\mathbb{R}^{d}} \tilde{H}(\mathbf{k}) d \mathbf{k}$ implies that $\tilde{H}(\mathbf{k}) \leq 1$, i.e.,

$$
0 \leq \tilde{H}(\mathbf{k}) \leq 1 \text { for all } \mathbf{k} .
$$

It follows that any positive semidefinite Hermitian-symmetric kernel $H(\mathbf{r})=H(-\mathbf{r})$ whose Fourier transform satisfies the inequalities in (26) describes a determinantal point process with a pair correlation function given by

$$
g_{2}(\mathbf{r})=1-|H(\mathbf{r})|^{2},
$$

such that

$$
0 \leq g_{2}(\mathbf{r}) \leq 1 \quad \text { and } \quad g_{2}(\mathbf{0})=0,
$$

and a $n$-particle density given by (25). We see that the total correlation function for a determinantal point process is given by

$$
h(\mathbf{r})=-|H(\mathbf{r})|^{2} .
$$

The fact that the $n$-particle density functions can be written in terms of the determinant specified by (25) leads to bounds on $\rho_{n}$. For example, it trivially follows 
that

$$
\rho_{n}\left(\mathbf{r}_{12}, \mathbf{r}_{13}, \ldots, \mathbf{r}_{1 n}\right) \leq 1
$$

A less obvious but stronger upper bound is as follows:

$$
\rho_{n}\left(\mathbf{r}_{12}, \mathbf{r}_{13}, \ldots, \mathbf{r}_{1 n}\right) \leq \rho_{2}\left(\mathbf{r}_{12}\right) \rho_{2}\left(\mathbf{r}_{13}\right) \cdots \rho_{2}\left(\mathbf{r}_{1 n}\right) .
$$

We remark also that:

$$
\rho_{n}\left(\mathbf{r}_{12}, \ldots, \mathbf{r}_{1 n}\right) \leq \rho_{m}\left(\mathbf{r}_{12}, \ldots, \mathbf{r}_{1 m}\right) \quad \forall m \leq n .
$$

Each of these inequalities is a consequence of the determinantal form for $\rho_{n}$ and the characteristics of $H$; in particular, (32) follows directly from Fischer's inequality [32] and an appropriate partition of the matrix representation for the operator $\mathcal{H}$. Equation (31) is a result of the more general Hadamard-Fischer inequalities [32] and the relation $H(\mathbf{0})=1$. The positive semidefinite character of $\mathcal{H}$ is essential for these inequalities to hold.

A unique property of determinantal point processes is that each of the $n$-particle correlation functions $g_{n}$ can be expressed completely in terms of the pair correlation function $g_{2}$. Namely, at unit density we may write:

$$
\begin{aligned}
& g_{2}(r)=1-[H(r)]^{2} \\
& \Rightarrow H(r)= \pm \sqrt{1-g_{2}(r)} .
\end{aligned}
$$

Therefore:

$$
\begin{aligned}
g_{n}\left(\mathbf{r}_{12}, \ldots, \mathbf{r}_{1 n}\right) & =\operatorname{det}\left[H\left(r_{i j}\right)\right]_{1 \leq i<j \leq n} \\
& =\operatorname{det}\left[ \pm \sqrt{1-g_{2}\left(r_{i j}\right)}\right]_{1 \leq i<j \leq n} .
\end{aligned}
$$

The right side of (34) is well-defined for all $r \in \mathbb{R}^{+}$since $0 \leq g_{2}(r) \leq 1 \forall r$. We note that in general $H(r)$ may be either positive or negative for a given value of $r$ as in (2); therefore, the sign of the square root in (34) must be chosen appropriately. Our ability to express each $g_{n}$ in terms of the pair correlation function $g_{2}$ is a reflection of the fact that the $n$-particle correlation functions depend on a common kernel $H$; such a reformulation is generally not possible for an arbitrary point process. Thus, one can infer the behavior of the $n$-particle correlation functions for $n \geq 3$ solely from a knowledge of the behavior of $g_{2}$.

A trivial example of a determinantal point process is the case in which $H(0)=1$ and $H(r)=0$ for $r \neq 0$. The resulting pair correlation function is given by $g_{2}(0)=0$ and $g_{2}(r)=1 \forall r \neq 0$, and this function belongs to the same equivalence class as the pair correlation function of the Poisson point process with respect to Lebesgue measure on the nonnegative reals $\mathbb{R}^{+}$(i.e., the functions differ only on a set of measure zero). Note that Costin and Lebowitz [35] have considered the conditions under which a pair correlation function of the form $g_{2}(r)=1-\exp (-\lambda r)$ is a determinantal point process in $\mathbb{R}^{d}$.

We note in passing that the number of points in a determinantal point process that falls in a compact set $A \subset \mathbb{R}^{d}$ has the same distribution as a sum of independent 
$\operatorname{Bernoulli}\left(\lambda_{i}^{A}\right)$ random variables, where $\lambda_{i}^{A}$ are the eigenvalues of the operator $\mathcal{H}$ [29]. Moreover, Hough et al. [29] presented an algorithm to generate determinantal point process in $\mathbb{R}^{d}$, which we apply elsewhere [14].

\section{New Determinantal Point Processes in $\mathbb{R}^{d}$ and Their Connection to Fermionic Gases}

\section{1. "Fermi-Sphere" Point Processes in $\mathbb{R}^{d}$}

Here we obtain the appropriate generalization of (11) that corresponds to a determinantal point process in $\mathbb{R}^{d}$. First, we make the simple observation that the pair correlation function specified by (11) is related to the Fourier transform $\tilde{\alpha}(k ; R)$ of the onedimensional scaled intersection volume evaluated at $k=r$ and $R=\pi$, namely,

$$
g_{2}(r)=1-\frac{\tilde{\alpha}(r ; \pi)}{2 \pi}
$$

where $\tilde{\alpha}(k ; R)$ is given by (24) for $d=1$. A natural generalization of this pair correlation function in $\mathbb{R}^{d}$ is to replace the one-dimensional Fourier transform in (36) with its $d$ dimensional counterpart (24) evaluated at $k=r$ and divided by $(2 \pi)^{d}$, i.e.,

$$
g_{2}(r)=1-\frac{\tilde{\alpha}(r ; R)}{(2 \pi)^{d}} .
$$

However, the value of $R$ in each dimension must be chosen so that such a pair correlation function corresponds to a determinantal point process in that dimension. In other words, if we take the positive semidefinite function $H(\mathbf{r})$ to be given by the following radial function:

$$
H(r)=\frac{\sqrt{\tilde{\alpha}(r ; R)}}{(2 \pi)^{d / 2}}=\frac{\sqrt{\Gamma(1+d / 2)}}{\pi^{d / 4}} \frac{J_{d / 2}(r R)}{r^{d / 2}},
$$

$R$ must be determined so that the conditions (26) and (28) are satisfied. Noting that the expansion of $|H(r)|^{2}$ for small $r$ to leading order is given by

$$
|H(r)|^{2}=\frac{R^{d}}{2^{d} \pi^{d / 2} \Gamma(1+d / 2)}+\mathcal{O}\left(r^{2}\right)
$$

and using the condition that $g_{2}(0)=0[\mathrm{cf}$. (28) $]$ yields that

$$
R=K \equiv 2 \sqrt{\pi}[\Gamma(1+d / 2)]^{1 / d} .
$$

Now we must show that a pair correlation function (37) with $R=K$ satisfies the bounds of (28) and the bounds (26) on the spectral function $\tilde{H}(k)$. The square of the function $H(r)$, specified by (38), is clearly positive and achieves its maximum value of unity at the origin when $R=K$ and tends to zero in the limit $r \rightarrow \infty$, and hence the bounds of (28) are satisfied when $R=K$. Referring to relation (23) for the Fourier transform of the window indicator function, we see that the Fourier transform of (38) with $R=K$ is simply the indicator function

$$
\tilde{H}(k)=w(k ; K)=\Theta(K-k),
$$


which automatically satisfies (26), where $w$ is specified by (16) and $K$ is given by (40).

In summary, we have demonstrated that there is a determinantal point process in $\mathbb{R}^{d}$ with $n$-particle densities given by (25) with the kernel

$$
H(r)=\frac{\sqrt{\tilde{\alpha}(r ; K)}}{(2 \pi)^{d / 2}},
$$

where $K$ is given by (40). We will call such a determinantal point process a "Fermisphere" point process because $\tilde{H}(k)$ corresponds to a spherical window indicator function in Fourier space and, as we will see, corresponds to the ground state of a fermionic system in which the Fermi sphere is completely filled (see Section 3). In particular, the pair correlation function of such a point process [33] is given by

$$
g_{2}(r)=1-2^{d} \Gamma(1+d / 2)^{2} \frac{J_{d / 2}^{2}(K r)}{(K r)^{d}} .
$$

Moreover, the corresponding structure factor, at unit number density, takes the form

$$
S(k)=1-\alpha(k ; K),
$$

where $\alpha(k ; K)$ is the scaled intersection volume of two $d$-dimensional spheres of radius $K$ separated by a distance $k$, i.e., the function $\alpha(r ; R)$, specified by (21), with the replacements $r \rightarrow k$ and $R \rightarrow K$. Relation (44) is easily obtained by taking the Fourier transform of the total correlation function defined by (29), where $H(r)$ is given by (42), and employing the definition (7) for the structure factor. It follows from the properties of $\alpha(k ; K)$ in (44) that the structure factor $S(k)$ obeys the following bounds for all $k$ :

$$
0 \leq S(k) \leq 1
$$

and achieves its maximum value of unity for $k \geq 2 K$. The corresponding cumulative coordination number is given by

$$
Z(r)=v_{1}(r)-d \int_{0}^{r} \frac{J_{d / 2}^{2}(K x)}{x} \mathrm{~d} x .
$$

Note that the first term in (46) is precisely the cumulative coordination number for a Poisson point process in $\mathbb{R}^{d}$ and that the second term is strictly negative for any $r>0$, which is a reflection of the short-range repulsive nature of the point process. We will show that for sufficiently large $r$, the dominant contribution to $Z(r)$ will be the Poissonian term $v(r)$. We will see it is the cumulative coordination number $Z(r)$, rather than the pair correlation function (contrary to conventional wisdom), that enables one to identify and quantify an effective "hard-core" diameter of the determinantal point processes.

It is instructive to examine the asymptotic behaviors of $g_{2}(r), S(k)$, and $Z(r)$ for the Fermi-sphere point process. By virtue of the asymptotic properties of the Bessel function of arbitrary order, the small- $r$ and large- $r$ forms of the pair correlation function (43) are respectively given by

$$
g_{2}(r)=\frac{K^{2}}{d+2} r^{2}-\frac{(d+3) K^{4}}{2(d+2)^{2}(d+4)} r^{4}+\mathcal{O}\left(r^{6}\right) \quad(r \rightarrow 0)
$$


and

$$
g_{2}(r)=1-\frac{2 \Gamma(1+d / 2) \cos ^{2}(r K-\pi(d+1) / 4)}{K \pi^{d / 2+1} r^{d+1}} \quad(r \rightarrow \infty) .
$$

We see that $g_{2}(r)$ tends to zero quadratically in $r$ in the limit $r \rightarrow 0$, independent of the dimension. The coefficient of the quadratic term in (47) for positive $d$ attains it maximum value of $\pi^{2} / 3=3.2898 \ldots$ for $d=1$ and monotonically decreases with increasing dimension, asymptoting to $2 \pi / e=2.3114 \ldots$ in the limit $d \rightarrow \infty$. Moreover, $g_{2}(r)$ tends to unity for large pair distances with a decay rate that is controlled by the power law $1 / r^{d+1}$ for any $d \geq 1$.

The latter result implies that the structure factor $S(k)$ tends to zero linearly in $k$ in the limit $k \rightarrow 0$. Using (21) and (44), it is easy to verify that as $k$ tends to zero at $\rho=1$

$$
S(k)=\frac{c(d)}{2 K} k+\mathcal{O}\left(k^{3}\right) \quad(k \rightarrow 0),
$$

where $c(d)$ is a $d$-dependent positive constant given by (20). We see that at unit density, the point process in $\mathbb{R}^{d}$ for any $d$ is hyperuniform, and the structure factor $S(k)$ has a nonanalytic behavior at the origin given by $S(k) \sim|k|(k \rightarrow 0)$. The coefficient of the linear term in (49) for positive $d$ attains it minimum value of $1 /(2 \pi)=0.1591 \ldots$ for $d=1$ and monotonically increases with increasing dimension such that it asymptotes to $e^{1 / 2} /(2 \pi)=0.2624 \ldots$ in the limit $d \rightarrow \infty$. In three dimensions, this unusual linear nonanalytic behavior of the structure factor at $k=0$ is a well-known property of bosons in their grounds states [10, 11, 12] and, more recently, has been shown to characterize maximally random jammed sphere packings [13].

In Fig. 3, we compare pair statistics in both real and Fourier space for $d=1$ and $d=3$ at unit density. We see that the amplitudes of the oscillations in $g_{2}(r)$ that are apparent for $d=1$ are significantly reduced in the corresponding three-dimensional pair correlation function. The smallest value of $r$ for which $g_{2}(r)$ attains its maximum value of unity, which we denote by $r_{0}$, is determined by the first positive zero of $J_{d / 2}(K r)$, which for sufficiently large $d$ is given by

$$
K r_{0}=\frac{d}{2}+1.4729154 d^{1 / 3}+\frac{1.301687}{d^{1 / 3}}-\frac{0.007942}{d}+\mathcal{O}\left(\frac{1}{d^{5 / 3}}\right) \quad(d \rightarrow \infty) .
$$

Since $K$ increases with increasing $d$ and grows like $\sqrt{2 \pi d / e}$ for large $d, r_{0}$ grows like the square root of $d$ for large $d$. Similarly, the smallest value of $k$ for which $S(k)$ attains its maximum value of unity grows with increasing $d$ and for large $d$ grows like $\sqrt{d}$.

The results immediately above enable us to obtain the following small- $r$ and large- $r$ forms of the cumulative coordination number:

$$
Z(r)=v_{1}(r)\left[\frac{d K^{2}}{(d+2)^{2}} r^{2}-\frac{d(d+3) K^{4}}{2(d+2)^{2}(d+4)^{2}} r^{4}+\mathcal{O}\left(r^{6}\right)\right] \quad(r \rightarrow 0)
$$

and

$$
Z(r)=v_{1}(r)-1+\mathcal{O}\left(r^{-1}\right) \quad(r \rightarrow \infty)
$$



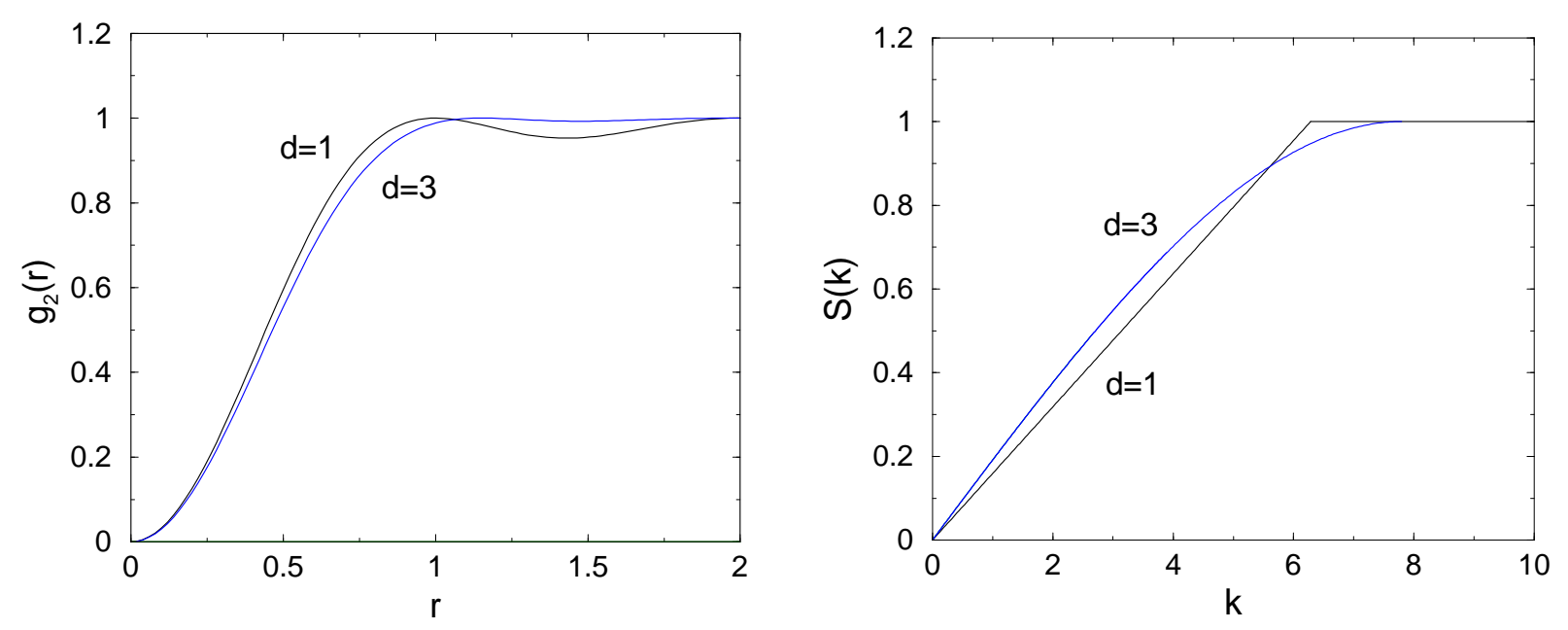

Figure 3. Left panel: Comparison of the pair correlation functions for $d=1$ and $d=3$. Right panel: The corresponding structure factors.

It is straightforward to prove that the asymptotic result (52) is a rigorous lower bound on $Z(r)$ for all $r$ using the identity $\int_{0}^{\infty} d x J_{d / 2}^{2}(x K) / x=1 / d$, the inequality $\int_{0}^{\infty} d x J_{d / 2}^{2}(x K) / x \geq \int_{0}^{r} d x J_{d / 2}^{2}(x K) / x$ for all positive $r$, and relation (46). Indeed, since $Z(r)$ is always nonnegative, we have the lower bounds

$$
Z(r) \geq\left\{\begin{array}{l}
0, \quad r<D, \\
v_{1}(r)-1, \quad r \geq D,
\end{array}\right.
$$

where the length scale

$$
D=\frac{\Gamma(1+d / 2)^{1 / d}}{\sqrt{\pi}}
$$

is the zero of $v_{1}(r)-1$. The length scale $D$ can be regarded as an upper bound on the effective hard-core diameter, which clearly grows with increasing dimension as vividly illustrated in Fig. 4, which compares the exact form of $Z(r)$ with the lower bound (53) for $d=3$ and $d=17$. For large $d$, the effective hard-core diameter is given by the asymptotic expression

$$
D=\sqrt{\frac{d}{2 \pi e}}\left[1+\frac{\ln (d)}{2}+\mathcal{O}(1)\right] \quad(d \rightarrow \infty),
$$

which is seen to grow like the square root of $d$. This growth of the effective hardcore diameter with dimension is a conceptually important conclusion that runs counter to conventional understanding of corresponding fermionic systems, which identifies the inverse "Fermi" radius, i.e., $K^{-1}=1 /\left[2 \sqrt{\pi} \Gamma(1+1 / d)^{1 / d}\right]$ (a decreasing function of $d$ ) with an effective hard-core diameter. We elaborate on this point in Section 3.

The hyperuniformity of the point process implies that the number variance $\sigma^{2}(R)$ for a spherical window of radius $R$ must grow slower than $R^{d}$ (i.e., the window volume) for large $R$. However, the fact that the large- $r$ behavior of the pair correlation function is controlled by the power law $1 / r^{d+1}$ means that $\sigma^{2}(R)$ must also grow faster than the 


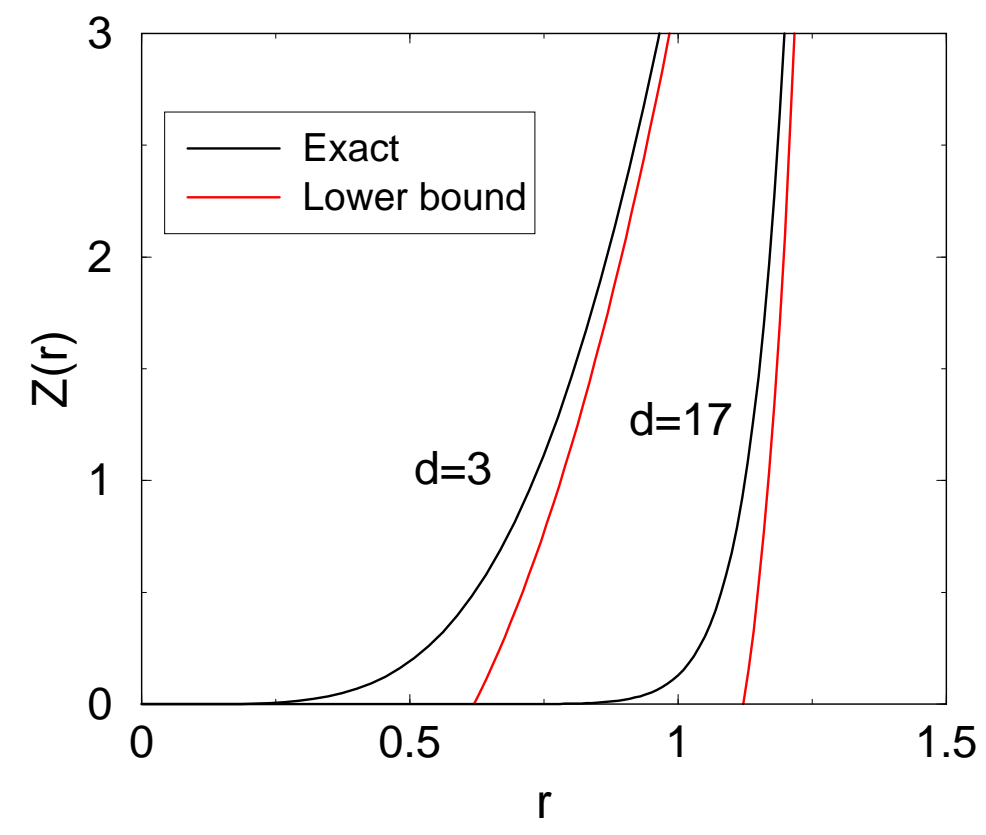

Figure 4. Cumulative coordination number $Z(r)$ as a function of $r$ for $d=3$ and $d=17$ compared to the corresponding lower bounds obtained from the inequality (53).

surface area of the window or $R^{d-1}$. In particular, upon substitution of (43) into (13), an asymptotic analysis reveals that for large $R$

$$
\begin{gathered}
\sigma^{2}(R)=\left\{\frac{d \pi^{(d-4) / 2}}{2 \Gamma[(d+1) / 2] \Gamma(1+d / 2)^{1 / d}} \ln (R)+C(d)\right\} R^{d-1} \\
+\mathcal{O}\left(R^{d-2}\right) \quad(R \rightarrow \infty),
\end{gathered}
$$

where $C(d)$ is a $d$-dimensional constant of order unity. We remark that a similar asymptotic scaling is expected to hold even when the observation window is nonspherical; a discussion of this point has been provided in [34]. We see that the number variance scaled by the window surface area, $\sigma^{2}(R) / R^{d-1}$, grows like $\ln (R)$, independent of the dimension. This unusual number variance growth law in three dimensions has also been recently seen in maximally random jammed sphere packings [13], which can be viewed as prototypical glasses because they are simultaneously perfectly rigid mechanically and maximally disordered. Note that the coefficient multiplying $\ln (R)$ in (56) decays to zero exponentially fast as $d \rightarrow \infty$, and, therefore, the surface-area term $R^{d-1}$ increasingly becomes the dominant one in the large- $d$ limit. This behavior should be contrasted with number variance for a Poisson point process, which grows like the window volume, i.e., $R^{d}$.

Figure 5 graphically depicts the Fermi-sphere point processes in one and two dimensions, which are generated using the algorithm of Hough et. al. [29]. Details and applications of this algorithm are reported by us elsewhere [14]. 

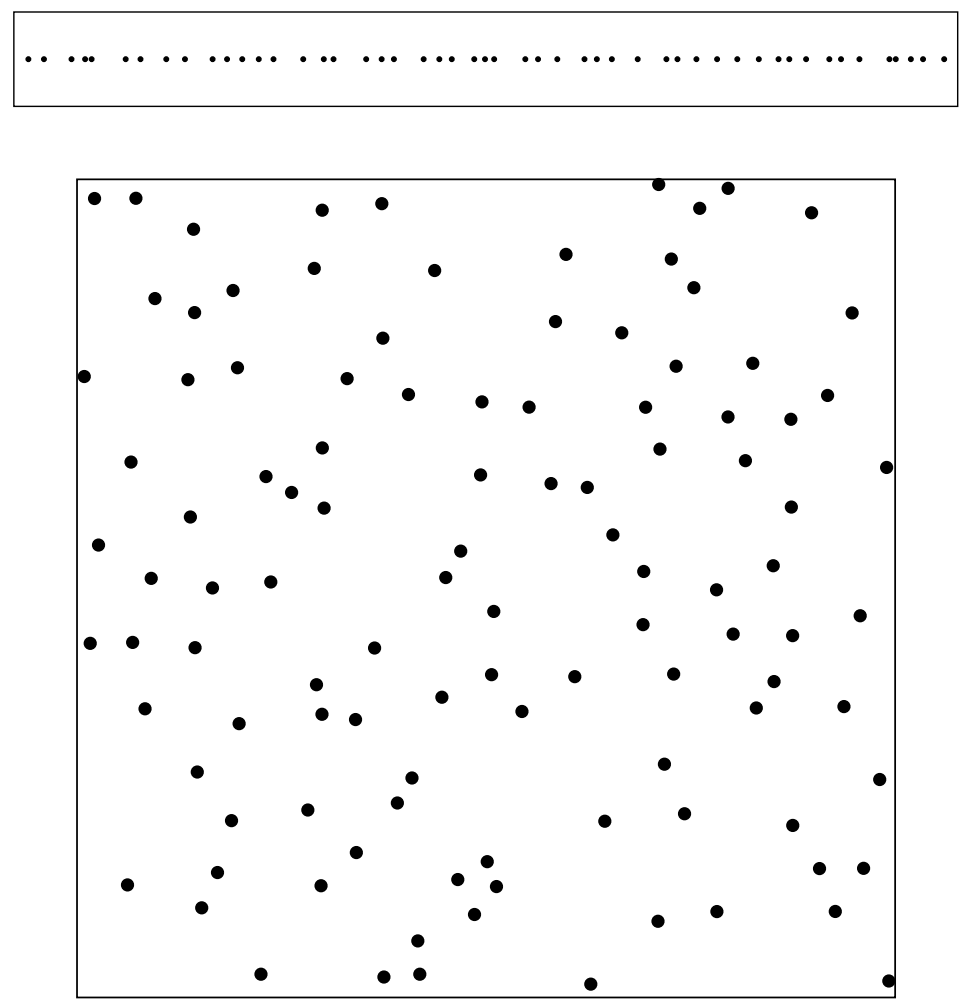

Figure 5. Top panel: A realization of 50 points of a Fermi-sphere point process in a linear "box" subject to periodic boundary conditions. Bottom panel: A realization of 100 points of a Fermi-sphere point process in a square box subject to periodic boundary conditions.

\section{2. "Fermi-Shells" Point Processes in $\mathbb{R}^{d}$}

Here we consider a generalization of the Fermi-sphere point process in which $\tilde{H}(k)$ is an indicator function for concentric rings in reciprocal space; we denote the resulting determinantal process as the "Fermi-shells" point process. Without loss of generality, define $2 m$ radii $k_{F}^{(j)}$, where $j \in\{1,2, \ldots, 2 m-1,2 m\}$ and $m \in \mathbb{N}$, such that the region of $d$-dimensional space within the ball $B\left(0 ; k_{F}^{(1)}\right)$ is empty, the annulus between $k_{F}^{(2)}$ and $k_{F}^{(1)}$ is filled, and so forth. We therefore have $m$ filled concentric rings in reciprocal space. Since the conditions for a determinantal point process are fulfilled for any indicator function in reciprocal space [35], the pair correlation function will still be given by (27). The calculation of $H(r)$ proceeds as follows:

$$
H(r)=\left(\frac{1}{2 \pi}\right)^{d} \sum_{j=1}^{m}\left[\mathfrak{F}\left\{\Theta\left(k_{F}^{(2 j)}-k\right)\right\}-\mathfrak{F}\left\{\Theta\left(k_{F}^{(2 j-1)}-k\right)\right\}\right],
$$

which implies:

$H(r)=\left(\frac{1}{2 \pi}\right)^{d / 2} \sum_{j=1}^{m}\left[\left(\frac{k_{F}^{(2 j)}}{r}\right)^{d / 2} J_{d / 2}\left(k_{F}^{(2 j)} r\right)-\left(\frac{k_{F}^{(2 j-1)}}{r}\right)^{d / 2} J_{d / 2}\left(k_{F}^{(2 j-1)} r\right)\right]$,

where $\mathfrak{F}$ denotes the Fourier transform to coordinate space. 
It is important to note that the values of the various $k_{F}^{(j)}$ are not independent of each other and are constrained by the density (here set to unity) according to:

$$
\sum_{j=1}^{m}\left[\left(k_{F}^{(2 j)}\right)^{d}-\left(k_{F}^{(2 j-1)}\right)^{d}\right]=(2 \sqrt{\pi})^{d} \Gamma(1+d / 2) .
$$

The filling of Fermi shells generally introduces a greater level of short-range correlations relative to the Fermi-sphere case (see Fig. 6).

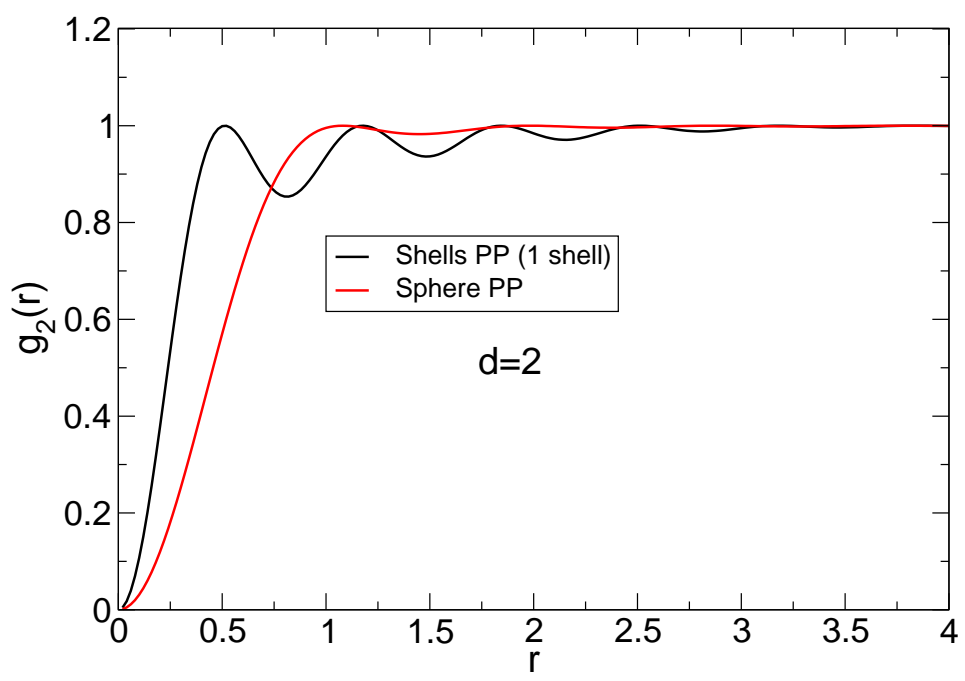

Figure 6. Comparison of the pair correlation function of the Fermi-sphere point process $(\mathrm{PP})$ to that of the Fermi-shells point process for $d=2$. In the latter case, $k_{F}=2 \sqrt{\pi}, k_{F}^{(1)}=4$ and $k_{F}^{(2)}=\sqrt{k_{F}^{2}+\left(k_{F}^{(1)}\right)^{2}}$.

\subsection{Spin-Polarized Fermionic Gas in $\mathbb{R}^{d}$}

Note that we do not know of any correspondence of the general Fermi-shells point process in $\mathbb{R}^{d}$ for any $d \geq 2$ to random matrix theory or the zeros of any generalized zeta function in number theory. However, we can show that a spin-polarized fermionic gas in $\mathbb{R}^{d}$ for any $d \geq 1$ has the same $n$-particle densities. For simplicity, this comparison will be done for the Fermi-sphere case, i.e., we will demonstrate that the $\rho_{n}$ are given by (25) with $H(r)$ given by (42) provided that the Fermi radius $k_{F}=K=2 \sqrt{\pi} \Gamma(1+d / 2)^{1 / d}$.

We first recall some general properties of the $n$-particle density functions $\rho_{n}$ for a ground-state noninteracting gas of fermions in $\mathbb{R}^{d}, d \geq 1$. Assume that we have $N$ spin-polarized fermions in a volume $V$ with number density $\rho=N / V$. The $n$-particle density functions are defined for all $n \leq N$ with respect to the ground state $\left|\phi_{0}\right\rangle$ by:

$$
\rho_{n}\left(\mathbf{x}_{1}, \ldots, \mathbf{x}_{n}\right)=\left\langle\phi_{0}\left|\prod_{i=1}^{n}\left[\psi^{*}\left(\mathbf{x}_{i}\right) \psi\left(\mathbf{x}_{i}\right)\right]\right| \phi_{0}\right\rangle,
$$

where the operators $\psi^{*}(\mathbf{x}), \psi(\mathbf{x})$ are the creation and annihilation field operators, respectively. Using the momentum representation, the field operators are defined in 
terms of the particle creation and annihilation operators $a_{\mathbf{k}}^{*}, a_{\mathbf{k}}$ :

$$
\begin{aligned}
& \psi(\mathbf{x})=\sum_{\mathbf{k}} \phi_{\mathbf{k}}(\mathbf{x}) a_{\mathbf{k}} \\
& \psi^{*}(\mathbf{x})=\sum_{\mathbf{k}} \overline{\phi_{\mathbf{k}}(\mathbf{x})} a_{\mathbf{k}}^{*} \\
& \phi_{\mathbf{k}}(\mathbf{x})=\left(\frac{1}{V}\right)^{1 / 2} \exp [\mathrm{i}(\mathbf{k}, \mathbf{x})] \\
& \overline{\phi_{\mathbf{k}}(\mathbf{x})}=\left(\frac{1}{V}\right)^{1 / 2} \exp [-\mathrm{i}(\mathbf{k}, \mathbf{x})],
\end{aligned}
$$

where $(\mathbf{k}, \mathbf{x})=\sum_{i=1}^{d} k_{i} x_{i}$ is the Euclidean inner product of two real-valued vectors. The field operators for fermions must satisfy the following anticommutation relation:

$$
\left\{\psi\left(\mathbf{x}_{i}\right), \psi^{*}\left(\mathbf{x}_{j}\right)\right\}=\delta\left(\mathbf{x}_{i}-\mathbf{x}_{j}\right) .
$$

Wick's Theorem along with the anticommutation relation (65) immediately allow us to write the expectation value in (60) as a determinant (equivalently, see Macchi's discussion of fermion processes [27]):

$$
\begin{aligned}
\rho_{n}\left(\mathbf{x}_{1}, \ldots, \mathbf{x}_{n}\right) & =\sum_{\sigma \in S_{n}}(-1)^{\sigma} \prod_{i=1}^{n}\left\langle\phi_{0}\left|\psi^{*}\left(\mathbf{x}_{i}\right) \psi\left(\mathbf{x}_{\sigma(i)}\right)\right| \phi_{0}\right\rangle \\
& =\operatorname{det}\left[\left\langle\phi_{0}\left|\psi^{*}\left(\mathbf{x}_{i}\right) \psi\left(\mathbf{x}_{j}\right)\right| \phi_{0}\right\rangle\right]_{1 \leq i, j \leq n},
\end{aligned}
$$

where $S_{n}$ denotes the permutation group for $n$ objects. Namely, Wick's Theorem relates the $n$-particle density functions to the $n$ ! ways of pairing the $2 n$ creation and annihilation field operators; anticommutation of the operators introduces the factor of $(-1)^{\sigma}$ in (66).

For the ground-state system, the argument of the determinant in (67) may be evaluated by filling the Fermi sphere up to the Fermi radius $k_{F}$ with the result:

$$
G\left(\mathbf{x}, \mathbf{x}^{\prime}\right)=\left\langle\phi_{0}\left|\psi^{*}(\mathbf{x}) \psi\left(\mathbf{x}^{\prime}\right)\right| \phi_{0}\right\rangle=\left(\frac{k_{F}}{2 \pi}\right)^{d / 2} r^{-d / 2} J_{d / 2}\left(k_{F}\left|\mathbf{x}-\mathbf{x}^{\prime}\right|\right) .
$$

Since $G$ is a translationally-invariant function, we will abuse notation slightly and write $G\left(\mathbf{x}, \mathbf{x}^{\prime}\right)=G\left(\left|\mathbf{x}-\mathbf{x}^{\prime}\right|\right)=G\left(r ; k_{F}\right)$, making the parameterization by $k_{F}$ explicit.

In accordance with our established convention and without loss of generality, let $\rho=1$. For a system of $N$ spin-polarized fermions, the Fermi radius $k_{F}$ is given exactly by:

$$
k_{F}=2 \sqrt{\pi}\{\Gamma[(d / 2)+1]\}^{1 / d}=K .
$$

Therefore, we may equivalently write for (68):

$$
G(r ; K)=\pi^{-d / 4} \sqrt{\Gamma[(d / 2)+1]} r^{-d / 2} J_{d / 2}(r K)=H(r) .
$$

The result in (70) shows that our proposed Fermi-sphere point process corresponds exactly to the one generated by a system of noninteracting fermions in $d$ dimensions.

The connection for $d=1$ between this system of noninteracting fermions and the CUE of random matrix theory implies that the correlations in the ground state resulting from the Pauli exclusion principle are equivalent to those induced on a $d=2$ Coulomb 
gas constrained to the unit ring and interacting via a logarithmic pair potential at finite temperature $T=1 / 2$ in units such that Boltzmann's constant $k_{B}=1$. The argument in Appendix A shows that this reduction of the probability density to a classical particle system with at most two-body interactions is peculiar to the choice of the indicator function in reciprocal space; in general, one must include at least three-body interactions to describe the system appropriately.

We also mention the significance of (54) in defining an effective hard core on the system of noninteracting fermions in each dimension. This issue is equivalent to assigning an appropriate length scale for the widely-studied (see, e.g., [39]) Fermi correlation hole. An argument primarily due to Slater [40] suggests that the correlations which result from antisymmetry in the many-body wavefunction extend outward for a distance $r_{0} \lesssim k_{F}^{-1}$. The reasoning behind this choice of length scale relies on the introduction of an exchange hole into the system with approximate spherical symmetry and a radius determined by the de Broglie wavelength associated with the Fermi radius $k_{F}$. This notion has been quantified for interacting atomic systems by considering, for example, the difference of distribution functions derived from Hartree-Fock and Hartree wavefunctions [41], but the $k_{F}^{-1}$ scaling for the noninteracting case is still reported in modern texts [42].

However, we recall from (40) that as $d$ increases, the value of the Fermi radius also increases; in fact, $k_{F} \sim \sqrt{d}$ for sufficiently large $d$. This behavior implies that $k_{F}^{-1} \rightarrow 0$ as $d \rightarrow+\infty$, meaning that the effective hard core diameter would become negligibly small for sufficiently large $d$ if it were to scale as $k_{F}^{-1}$. This conclusion contradicts the fact that $g_{2}(r) \rightarrow 0$ pointwise as $d \rightarrow+\infty$ for all $r \in[0,+\infty)$. In other words, the range in $r$ over which one finds a small probability of finding two particles in close proximity increases with dimension, requiring a different means by which to quantify the extent of the effective hard core.

The definition of $Z(r)$ in (8) suggests instead that we take the value of $D$ in (54) as a measure of the effective hard core diameter. We note that since $Z(r)$ is a nonnegative monotonically increasing function of $r$ and, therefore, nonzero for some range in $r$ over which its lower bound is zero, $D$ must represent an upper bound to the effective hard core radius. This representation of the effective hard-core diameter is quantitatively well-defined for any dimension due to the inclusion of the $x^{d-1}$ factor under the integral in (8) from the surface area of the $d$-dimensional ball; it is this factor which appropriately rescales $g_{2}$ such that (53) represents a true lower bound on $Z(r)$ in any dimension by the argument above. It is also for this reason that neither $g_{2}(r)$ nor $S(k)$ alone are sufficient to define the effective hard-core diameter in a quantitative manner. In contrast to Slater's scaling of the effective hard core diameter, $D \sim k_{F}$ in any dimension by the definition of the Fermi radius, which is in accordance with the high-dimensional behavior of both $g_{2}$ and $k_{F}$. 


\section{Nearest-Neighbor Functions}

It is useful to characterize point processes by examining other quantities besides the $n$-particle correlation functions. One popular descriptor used in one dimension is the so-called "gap" distribution function $p(z)$ [3], which characterizes the spacing between the points. In the random matrix theory literature, this quantity often has erroneously and misleadingly been called the "nearest-neighbor-spacing" distribution because gaps to the right of some reference point are considered. However, $p(z)$ makes no distinction between gaps to the left or right of some reference point. The quantity $p(z) d z$ gives the probability of finding a gap (a line interval empty of points) of length between $z$ and $z+d z$. The function $p(z)$ is called the chord length probability density in the theory of random media [36, 37].

In the case of random matrix theory, there exist exact representations of $p(z)$ for the spacings of the eigenvalues in the GOE, GUE, and GSE, but they can only be determined numerically for general situations. A remarkably accurate approximation for the GOE in the infinitely-large matrix limit is the so-called Wigner surmise. The Wigner surmise has been generalized to any of the aforementioned ensembles as follows:

$$
p_{\beta}(z)=A_{\beta} z^{\beta} e^{-B_{\beta} z^{2}},
$$

where the parameters $A_{\beta}$ and $B_{\beta}$, which depend on reciprocal temperature $\beta$, are obtained from the normalization of both $p(z)$ and its first moment, or the average gap size $\langle z\rangle$. For the GOE, GUE, and GSE, $\beta=1,2$, and 4 , respectively, and $A_{1}=\pi / 2$, $B_{1}=\pi / 4, A_{2}=32 / \pi^{2}, B_{2}=4 / \pi$, and $A_{4}=262144 /\left(729 \pi^{2}\right), B_{4}=64 /(9 \pi)$.

For $d \geq 2$, the gap distribution function is strictly not a meaningful descriptor of point processes. The natural generalizations of $p(z)$ in higher dimensions are the nearest-neighbor functions [38, 37]. Nearest-neighbor functions describe the probability of finding the nearest point of a point process in $\mathbb{R}^{d}$ at some given distance from a reference point in the space. Such statistical quantities are called "void" or "particle" nearest-neighbor functions if the the reference point is an arbitrary point of the space or an actual point of the point process, respectively.

\subsection{Definitions}

First, we recall the definitions of the void and particle nearest-neighbor probability density functions $H_{V}(r)$ and $H_{P}(r)$, respectively:

$$
\begin{aligned}
& H_{V}(r) \mathrm{d} r=\text { Probability that a point of the point process lies at } \\
& \text { a distance between } r \text { and } r+\mathrm{d} r \text { from an arbitrary } \\
& \text { point in the space. } \\
& H_{P}(r) \mathrm{d} r=\text { Probability that a point of the point process lies at } \\
& \text { a distance between } r \text { and } r+\mathrm{d} r \text { from another point (73) } \\
& \text { of the point process. }
\end{aligned}
$$


It is useful to introduce the associated dimensionless "exclusion" probabilities $E_{V}(r)$ and $E_{P}(r)$ defined as follows:

$$
\begin{aligned}
E_{V}(r)= & \begin{array}{l}
\text { Probability of finding a spherical cavity of radius } r \\
\text { empty of any points in the point process. }
\end{array} \\
E_{P}(r)= & \text { Probability of finding a spherical cavity of radius } r \\
& \text { centered at an arbitrary point of the point process } \\
& \text { empty of any other points. }
\end{aligned}
$$

It follows that the exclusion probabilities are complementary cumulative distribution functions associated with the density functions and thus are related to the latter via

$$
E_{V}(r)=1-\int_{0}^{r} H_{V}(x) \mathrm{d} x
$$

and

$$
E_{P}(r)=1-\int_{0}^{r} H_{P}(x) \mathrm{d} x .
$$

Differentiating the exclusion-probability relations with respect to $r$ gives

$$
H_{V}(r)=-\frac{\partial E_{V}}{\partial r}
$$

and

$$
H_{P}(r)=-\frac{\partial E_{P}}{\partial r}
$$

The $n$th moment of $H_{P}(r)$ is defined as

$$
\lambda_{n}=\int_{0}^{\infty} r^{n} H_{P}(r) \mathrm{d} r .
$$

Of particular interest to us is the mean nearest-neighbor distance

$$
\begin{aligned}
\lambda \equiv \lambda_{1} & =\int_{0}^{\infty} r H_{P}(r) \mathrm{d} r \\
& =\int_{0}^{\infty} E_{P}(r) \mathrm{d} r .
\end{aligned}
$$

It is useful to express the density functions $H_{V}(r)$ and $H_{P}(r)$ as a product of two functions as follows:

$$
H_{V}(r)=\rho s_{1}(r) G_{V}(r) E_{V}(r),
$$

and

$$
H_{P}(r)=\rho s_{1}(r) G_{P}(r) E_{P}(r),
$$

where $s_{1}(r)$ is the surface area of a $d$-dimensional sphere of radius $r$ given by (9). The quantities $G_{V}(r)$ and $G_{P}(r)$ are called the "conditional" nearest-neighbor functions and have the following interpretation:

$$
\begin{aligned}
\rho s_{1}(r) G_{V}(r) \mathrm{d} r= & \text { Given that a spherical cavity of radius } r \\
& \text { centered at an arbitrary point in the space is } \\
& \text { empty of any points of the point process, the } \\
& \text { probability of finding a point in the spherical } \\
& \text { shell of volume } s_{1}(r) \mathrm{d} r \text { surrounding the } \\
& \text { arbitrary point. }
\end{aligned}
$$


Point processes in arbitrary dimension

$$
\begin{aligned}
\rho s_{1}(r) G_{P}(r) \mathrm{d} r= & \text { Given that a spherical cavity of radius } r \\
& \text { centered at a randomly selected point of } \\
& \text { the point process is empty of any other } \\
& \text { points, the probability of finding a point } \\
& \text { in the spherical shell of volume } s_{1}(r) \mathrm{d} r \\
& \text { surrounding the randomly selected point. }
\end{aligned}
$$

Thus, it follows that the exclusion probabilities are also given by

$$
E_{V}(r)=\exp \left[-\rho s_{1}(1) \int_{0}^{r} x^{d-1} G_{V}(x) \mathrm{d} x\right]
$$

and

$$
E_{P}(r)=\exp \left[-\rho s_{1}(1) \int_{0}^{r} x^{d-1} G_{P}(x) \mathrm{d} x\right] .
$$

We remark that knowledge of any one function $H, E$, or $G$ (either void or particle) is sufficient to determine the other two functions via the relations mentioned above.

\subsection{Series Representations}

The nearest-neighbor functions can be expressed as infinite series whose terms are integrals over the $n$-particle density functions [38, 37]. For example, the void and particle exclusion probability functions for a translationally invariant point process are respectively given by

$$
E_{V}(r)=1+\sum_{k=1}^{\infty}(-1)^{k} \frac{\rho^{k}}{k !} \int_{\mathbb{R}^{d}} g_{k}\left(\mathbf{r}^{k}\right) \prod_{j=1}^{k} \Theta\left(r-\left|\mathbf{x}-\mathbf{r}_{j}\right|\right) \mathrm{d} \mathbf{r}_{j}
$$

and

$$
E_{P}(r)=1+\sum_{k=1}^{\infty}(-1)^{k} \frac{\rho^{k}}{k !} \int_{\mathbb{R}^{d}} g_{k+1}\left(\mathbf{r}^{k+1}\right) \prod_{j=2}^{k+1} \Theta\left(r-r_{1 j}\right) \mathrm{d} \mathbf{r}_{j} .
$$

The corresponding series for $H_{V}(r)$ and $H_{P}(r)$ are obtained from the series above using (78) and (79).

In general, an exact evaluation of the aforementioned infinite series are not possible, except for simple processes such as the Poisson point process. In the latter instance, because $\rho_{n}=\rho^{n}$, both series (88) and (89) can be summed exactly to give

$$
E_{V}(r)=E_{P}(r)=\exp \left[-\rho v_{1}(r)\right]
$$

where $v_{1}(r)$ is the volume of a $d$-dimensional sphere of radius $r$ given by (14). Therefore, for a Poisson point process, we have from (78), (79), (82), and (83) that

$$
H_{V}(r)=H_{P}(r)=\rho s_{1}(r) \exp \left[-\rho v_{1}(r)\right], \quad G_{V}(r)=G_{P}(r)=1 .
$$

We see that there is no distinction between the void and particle quantities for the Poisson distribution, which is generally not the case for correlated point processes. 


\subsection{Rigorous Bounds}

Torquato has given rigorous upper and lower bounds on the so-called canonical n-point correlation function $H_{n}\left(\mathbf{x}^{m} ; \mathbf{x}^{p-m} ; \mathbf{r}^{q}\right.$ ) (with $n=p+q$ and $m \leq p$ ) for point processes in $\mathbb{R}^{d}$. Since the void and particle exclusion probabilities and nearest-neighbor probability density functions are just special cases of $H_{n}$, then we also have strict bounds on them for such models. Let $X$ represent either $E_{V}, H_{V}, E_{P}$, or $H_{P}$ and $X^{(k)}$ represent the $k$ th term of the series for these functions. Furthermore, let

$$
W^{\ell}=\sum_{k=0}^{\ell}(-1)^{k} X^{(k)}
$$

be the partial sum. Then it follows that for any of the exclusion probabilities or nearestneighbor probability density functions, we have the bounds

$$
\begin{array}{ll}
X \leq W^{\ell}, & \text { for } \ell \text { even } \\
X \geq W^{\ell}, & \text { for } \ell \text { odd. }
\end{array}
$$

Application of the aforementioned inequalities yield the first three successive bounds on the nonnegative void exclusion probability:

$$
\begin{aligned}
& E_{V}(r) \leq 1 \\
& E_{V}(r) \geq 1-\rho v_{1}(r) \\
& E_{V}(r) \leq 1-\rho v_{1}(r)+\frac{\rho^{2}}{2} s_{1}(1) \int_{0}^{2 r} x^{d-1} v_{2}^{i n t}(x ; r) g_{2}(x) \mathrm{d} x,
\end{aligned}
$$

where $v_{2}^{\text {int }}(x ; r)=v_{1}(r) \alpha(x ; r)$ is the intersection volume of two $d$-dimensional spheres (cf. (18)). The corresponding first two nontrivial bounds on the nonnegative nearestneighbor probability density function $H_{V}(r)$ are as follows:

$$
\begin{aligned}
& H_{V}(r) \leq \rho s_{1}(r) \\
& H_{V}(r) \geq \rho s_{1}(r)-\frac{\rho^{2}}{2} s_{1}(1) \int_{0}^{2 r} x^{d-1} s_{2}^{i n t}(x ; r) g_{2}(x) \mathrm{d} x,
\end{aligned}
$$

where $s_{2}^{\text {int }}(x ; r) \equiv \partial v_{2}^{\text {int }}(x ; r) / \partial r$ is the surface area of the intersection volume $v_{2}^{\text {int }}(x ; r)$. Bounds on the conditional function $G_{V}(r)$ follow by combining the bounds above on $E_{V}(r)$ and $H_{V}(r)$ and definition (82). For example, we obtain the following bounds

$$
G_{V}(r) \leq \frac{1}{1-\rho v_{1}(r)}
$$

and

$$
G_{V}(r) \geq \frac{1-\frac{\rho}{s_{1}(r)} s_{1}(1) \int_{0}^{2 r} x^{d-1} s_{2}^{i n t}(x ; r) g_{2}(x) \mathrm{d} x}{1-\rho v_{1}(r)+\frac{\rho^{2}}{2} s_{1}(1) \int_{0}^{2 r} x^{d-1} v_{2}^{i n t}(x ; r) g_{2}(x) \mathrm{d} x},
$$

which should only be applied for $r$ such that $G_{V}(r)$ remains positive. The bounds above lead to the following properties of the nearest-neighbor functions at the origin:

$$
E_{V}(0)=1, \quad H_{V}(0)=0, \quad G_{V}(0)=1 .
$$


Point processes in arbitrary dimension

Similarly, the first three successive bounds on the particle exclusion probability are given by

$$
\begin{aligned}
& E_{P}(r) \leq 1 \\
& E_{P}(r) \geq 1-Z(r) \\
& E_{P}(r) \leq 1-Z(r) \\
& +\frac{\rho^{2}}{2} \int_{\mathbb{R}^{d}} \int_{\mathbb{R}^{d}} \Theta\left(r-r_{12}\right) \Theta\left(r-r_{13}\right) g_{3}\left(r_{12}, r_{13}, r_{23}\right) \mathrm{d} \mathbf{r}_{2} \mathrm{~d} \mathbf{r}_{3},
\end{aligned}
$$

where $Z(r)$ is the cumulative coordination number defined by (8). The corresponding first two nontrivial bounds on the nonnegative nearest-neighbor probability density function $H_{P}(r)$ are as follows:

$$
\begin{aligned}
& H_{P}(r) \leq \rho s_{1}(r) g_{2}(r) \\
& H_{P}(r) \geq \rho s_{1}(r) g_{2}(r) \\
& -\rho^{2} \int_{\mathbb{R}^{d}} \int_{\mathbb{R}^{d}} \delta\left(r-r_{12}\right) \Theta\left(r-r_{13}\right) g_{3}\left(r_{12}, r_{13}, r_{23}\right) \mathrm{d} \mathbf{r}_{2} \mathrm{~d} \mathbf{r}_{3},
\end{aligned}
$$

where $\delta(r)$ is the radial Dirac delta function. Bounds on the conditional function $G_{P}(r)$ follow by combining the bounds on $E_{P}(r)$ and $H_{P}(r)$ and definition (83). For example, we obtain the following bounds:

$$
G_{P}(r) \leq \frac{g_{2}(r)}{1-Z(r)}
$$

and

$G_{P}(r) \geq \frac{g_{2}(r)-\frac{\rho}{s_{1}(r)} \int_{\mathbb{R}^{d}} \int_{\mathbb{R}^{d}} \delta\left(r-r_{12}\right) \Theta\left(r-r_{13}\right) g_{3}\left(r_{12}, r_{13}, r_{23}\right) \mathrm{d} \mathbf{r}_{2} \mathrm{~d} \mathbf{r}_{3}}{1-Z(r)+\frac{\rho^{2}}{2} \int_{\mathbb{R}^{d}} \int_{\mathbb{R}^{d}} \Theta\left(r-r_{12}\right) \Theta\left(r-r_{13}\right) g_{3}\left(r_{12}, r_{13}, r_{23}\right) \mathrm{d} \mathbf{r}_{2} \mathrm{~d} \mathbf{r}_{3}}$,

which should only be applied for $r$ such that $G_{P}(r)$ remains positive. The bounds above lead to the following properties of the nearest-neighbor functions at the origin:

$$
E_{P}(0)=1, \quad H_{P}(0)=0, \quad G_{P}(0)=0 .
$$

We now obtain bounds on the mean nearest-neighbor distance $\lambda$ at unit density using the aforementioned upper and lower bounds on $E_{P}(r)$. Let us define the following distances:

$$
\lambda_{L}=\int_{0}^{r_{0}}[1-Z(r)] \mathrm{d} r
$$

and

$$
\lambda_{U}=\int_{0}^{+\infty} \exp [-Z(r)] \mathrm{d} r
$$

where $r_{0}$ is the location of the zero of $1-Z(r)$. In light of the bounds (104) and (128), it is clear that $\lambda_{L}$ and $\lambda_{U}$ bound $\lambda$ from below and above, respectively, i.e.,

$$
\lambda_{L} \leq \lambda \leq \lambda_{U}
$$




\subsection{Results for Fermi-Sphere Point Processes}

5.4.1. Exact Determinantal Representations The Fermi-sphere point process is unique in that both the particle and void exclusion probabilities may be expressed as determinants over $N \times N$ matrices, the elements of which are related to overlap integrals of the basis functions $\phi_{\mathbf{k}}=(1 / \sqrt{V}) \exp [\mathrm{i}(\mathbf{k}, \mathbf{x})]$ on $B(\mathbf{0} ; r)$, a $d$-dimensional ball of radius $r$ centered at the origin (the exact location of the ball's center is irrelevant since the point process is translationally invariant). The thermodynamic limit can then be taken appropriately. We provide the details of this analysis elsewhere [14] and immediately state the results:

$$
\begin{aligned}
& E_{V}(r)=\operatorname{det}[\mathbb{I}-M(r)] \\
& E_{P}(r)=E_{V}(r) \operatorname{tr}\left\{A[\mathbb{I}-M(r)]^{-1}\right\},
\end{aligned}
$$

where $\mathbb{I}$ is the $N \times N$ identity matrix, and the matrices $M(r)$ and $A$ are defined by:

$$
\begin{aligned}
& M_{i j}(r)=\int_{B(\mathbf{0} ; r)} \overline{\phi_{i}(\mathbf{x})} \phi_{j}(\mathbf{x}) \mathrm{d} \mathbf{x} \\
& A_{i j}=\overline{\phi_{i}(\mathbf{0})} \phi_{j}(\mathbf{0}) / \rho,
\end{aligned}
$$

where $\rho$ is the number density. We recall that knowledge of $E_{V}(r)$ and $E_{P}(r)$ is sufficient to determine all of the remaining nearest-neighbor functions $H_{V / P}(r)$ and $G_{V / P}(r)$. Note that as $r \rightarrow 0, M_{i j}(r) \rightarrow 0$ for all $i$ and $j$, which provides the necessary results $E_{V}(0)=1$ and $E_{P}(0)=\operatorname{tr}(A)=\sum_{i}\left|\phi_{i}(\mathbf{0})\right|^{2} / \rho=H(\mathbf{0})=1$. We mention that these determinants must be evaluated numerically but essentially exactly for finite $N \times N$ matrices, where $N$ is chosen to be sufficiently large to capture accurately the behavior of the system in the thermodynamic limit. Evidence for this convergence is provided in another paper [14].

5.4.2. Bounds, Comparison to Exact Results, and Link to Sphere Packings We now obtain bounds for nearest-neighbor functions for the Fermi-sphere point process and compare them to the corresponding aforementioned exact results. Using the identity

$$
s_{1}(1) \int_{0}^{2 r} x^{d+1} v_{2}^{\text {int }}(x ; r) \mathrm{d} x=\frac{2 d}{d+2} r^{2}\left[v_{1}(r)\right]^{2},
$$

the leading order term of the small-r expansion (47), and the upper bound (97), we obtain the weaker upper bound

$$
E_{V}(r) \leq 1-\rho v_{1}(r)+\rho^{2} \frac{d K^{2}}{(d+2)^{2}} r^{2}\left[v_{1}(r)\right]^{2},
$$

which is exact through terms of order $r^{2(d+1)}$. Therefore, we also have

$$
H_{V}(r)=\rho s_{1}(r)-\rho^{2} \frac{2(d+1) K^{2}}{d(d+2)^{2}} r^{3}\left[s_{1}(r)\right]^{2}+\mathcal{O}\left(r^{2 d+3}\right)
$$

and

$$
G_{V}(r)=1+\rho v_{1}(r)+\rho^{2} v_{1}(r)^{2}+\mathcal{O}\left(r^{2 d+1}\right)
$$


We see that through order $r^{2 d+1}, G_{V}(r) \geq 1$. This bound will be shown to apply for any $r$.

Employing the inequality $g_{3}\left(r_{12}, r_{13}, r_{23}\right) \leq g_{2}\left(r_{12}\right) g_{2}\left(r_{13}\right)$ [cf. (31)] in the upper bound (105) on $E_{V}(r)$ and lower bound (107) on $H_{P}(r)$, we find the following corresponding weaker bounds:

$$
E_{P}(r) \leq 1-Z(r)+\frac{Z^{2}(r)}{2}
$$

and

$$
H_{P}(r) \geq \rho s_{1}(r) g_{2}(r)-\rho s_{1}(r) g_{2}(r) Z(r) .
$$

These bounds in conjunction with the analogous evaluations of the bounds (108) and (109) on the conditional pair function $G_{P}(r)$ yields its exact small- $r$ behavior up through terms of order $r^{4}$ :

$$
G_{P}(r)=\frac{K^{2}}{d+2} r^{2}-\frac{(d+3) K^{4}}{2(d+2)^{2}(d+4)} r^{4}+\mathcal{O}\left(r^{6}\right) .
$$

Comparing this expansion to (47) reveals that $G_{P}(r)=g_{2}(r)$ up through terms of order $r^{4}$.

We can also show that

$$
G_{V}(r) \geq 1 \quad \text { for all } r
$$

and

$$
G_{P}(r) \geq g_{2}(r) \quad \text { for all } r .
$$

These results are obtained from definitions (86) and (87) and the following upper bounds on the exclusion probabilities:

$$
E_{V}(r) \leq \exp \left[-\rho v_{1}(r)\right] \quad \text { for all } r
$$

and

$$
E_{P}(r) \leq \exp [-Z(r)] \quad \text { for all } r .
$$

To prove (127) and (128), we recall the series representations of $E_{V}$ and $E_{P}$ in (88) and (89), respectively, which we may rewrite in the following more compact form:

$$
\begin{aligned}
& E_{P / V}(r)=1+\sum_{k=1}^{+\infty}(-1)^{k} E_{P / V}^{(k)} \\
& E_{V}^{(k)}=\frac{\rho^{k}}{k !} \int_{\mathbb{R}^{d}} g_{k}\left(\mathbf{r}^{k}\right) \prod_{j=1}^{k} \Theta\left(r-\left|\mathbf{x}-\mathbf{r}_{j}\right|\right) \mathrm{d} \mathbf{r}_{j} \\
& E_{P}^{(k)}=\frac{\rho^{k}}{k !} \int_{\mathbb{R}^{d}} g_{k+1}\left(\mathbf{r}^{k}\right) \prod_{j=2}^{k+1} \Theta\left(r-r_{1 j}\right) \mathrm{d} \mathbf{r}_{j} .
\end{aligned}
$$

It is important to note that the series in (129) converge absolutely for all $r \in \mathbb{R}^{+}$, which is easily seen from the inequalities

$$
E_{V}^{(k)} \leq \frac{\rho^{k}}{k !} \int_{\mathbb{R}^{d}} \prod_{j=1}^{k} \Theta\left(r-\left|\mathbf{x}-\mathbf{r}_{j}\right|\right) \mathrm{d} \mathbf{r}_{j}=\frac{\left[\rho v_{1}(r)\right]^{k}}{k !}
$$


Point processes in arbitrary dimension

$$
E_{P}^{(k)} \leq \frac{\rho^{k}}{k !} \int_{\mathbb{R}^{d}} \prod_{j=2}^{k+1} g_{2}\left(r_{1 j}\right) \Theta\left(r-r_{1 j}\right) \mathrm{d} \mathbf{r}_{j}=\frac{[Z(r)]^{k}}{k !} .
$$

Equations (132) and (133) follow directly from the inequalities in (30) and (31), respectively. It is therefore true that

$$
\begin{aligned}
& \left|E_{V}(r)\right| \leq 1+\sum_{k=1}^{+\infty} E_{V}^{(k)} \leq \sum_{k=0}^{+\infty} \frac{\left[\rho v_{1}(r)\right]^{k}}{k !}=\exp \left[\rho v_{1}(r)\right]<+\infty \\
& \left|E_{P}(r)\right| \leq 1+\sum_{k=1}^{+\infty} E_{P}^{(k)} \leq \sum_{k=0}^{+\infty} \frac{[Z(r)]^{k}}{k !}=\exp [Z(r)]<+\infty
\end{aligned}
$$

and absolute convergence of the series in (129) implies convergence of those series, which in turn allows us to conclude that the sequences $E_{P / V}^{(k)} \rightarrow 0$ as $k \rightarrow+\infty$.

We now wish to compare the series in (129) with the following series representations of the proposed upper limits in (127) and (128):

$$
\begin{aligned}
& E_{V}^{(U L)}(r)=1-\rho v_{1}(r)+\sum_{k=2}^{+\infty} \frac{\left[-\rho v_{1}(r)\right]^{k}}{k !} \\
& E_{P}^{(U L)}(r)=1-Z(r)+\sum_{k=2}^{+\infty} \frac{[-Z(r)]^{k}}{k !} .
\end{aligned}
$$

Note that the series in (129), (136), and (137) agree up to their second terms; the alternating series test then implies that the contributions from the remaining terms is no greater than the magnitude of the third terms in the series. Equations (132) and (133) therefore show that the series in (136) and (137) in actuality do bound the series in (129) from above, thereby proving the claims. The lower bounds in (125) and (126) immediately follow from monotonicity and positivity of the exponential function along with (86) and (87). 

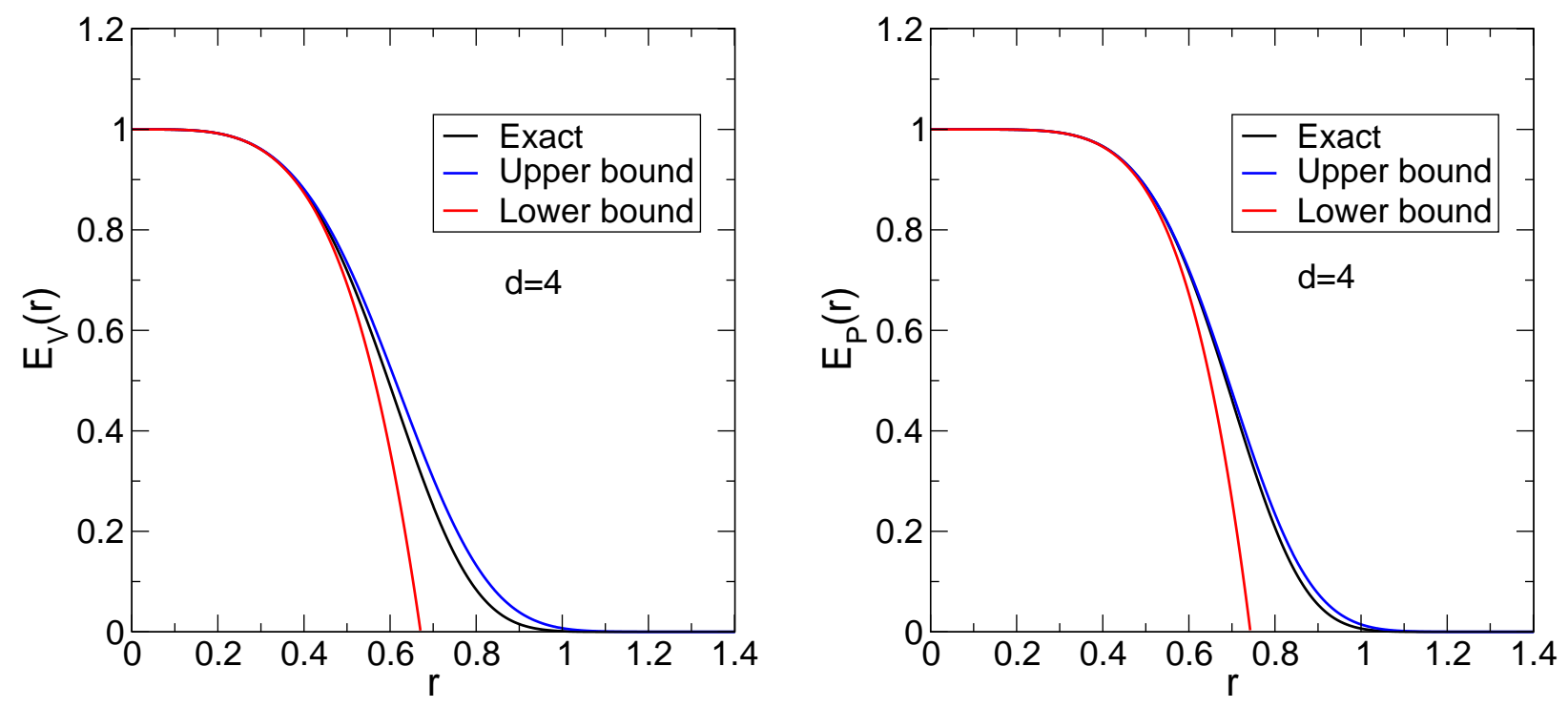

Figure 7. Left panel: Upper and lower bounds on $E_{V}(r)$ for $d=4$ and $\rho=1$ as obtained from (96) and (127) compared to the corresponding exact evaluation of it. Right panel: Upper and lower bounds on $E_{P}(r)$ for $d=4$ and $\rho=1$ as obtained from (104) and (128), respectively, compared to the corresponding exact evaluation of it.

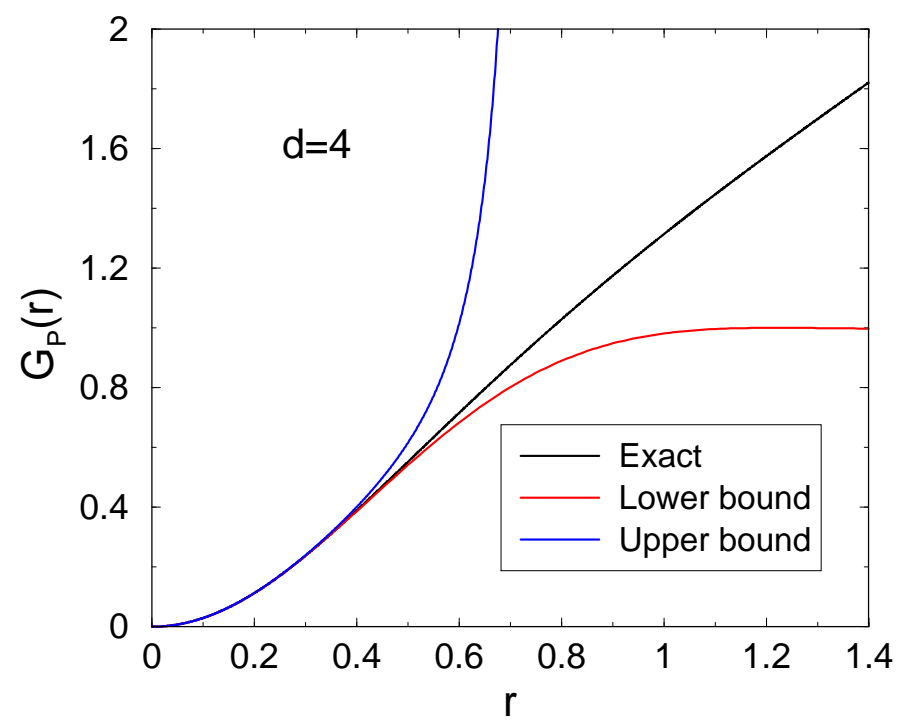

Figure 8. Upper and lower bounds on $G_{P}(r)$ for $d=4$ and $\rho=1$ as obtained from (104) and (128), respectively, compared to the corresponding exact evaluation of it.

The left panel of Fig. 7 compares the upper bound (127) and lower bound (96) of $E_{V}$ for a Fermi-sphere point process for $d=4$ to the corresponding exact evaluation. The right panel of the same figure compares the upper and lower bounds on $E_{P}(r)$ for $d=4$ as obtained from (104) and (128), respectively, to the corresponding exact evaluation of it. The upper bounds on both exclusion probabilities provide reasonable estimates of the exact results as compared to the corresponding lower bounds. Figure 8 
compares upper and lower bounds on $G_{P}(r)$ for $d=4$ as obtained from (104) and (128), respectively, to the corresponding exact evaluation of it. Not surprisingly, the bounds become better estimators as the dimension increases and therefore can be profitably used in high dimensions, where exact evaluations are difficult to obtain. The left panel of Fig. 9 shows the upper bound (127) and lower bound (96) for a Fermi-sphere point process for $d=17$. It is seen that the bounds essentially converge to unity for the range $0 \leq r \leq 0.8$ and are relatively close to one another for $0.8 \leq r \leq 1.1$. The right panel of Fig. 9 depicts the analogous bounds on $E_{P}(r)$ for $d=17$. Note that the bounds on $E_{V}(r)$ behave almost exactly like the bounds on $E_{P}(r)$ at this value of $d$. This graphically suggests that as $d$ becomes large, the exact expressions for $E_{V}(r)$ and $E_{P}(r)$ approach the same step function, which we demonstrate below.


Figure 9. Left panel: Upper and lower bounds on $E_{V}(r)$ for $d=17$ and $\rho=1$ as obtained from (96) and (127). Right panel: Upper and lower bounds on $E_{P}(r)$ for $d=17$ and $\rho=1$ as obtained from (104) and (128).

We now show that both $E_{P}$ and $E_{V}$ tend to the same step function $\Theta(D-r)$ in the large- $d$ limit, where $D$ is the estimate of the effective hard-core diameter defined by (54). We begin by utilizing the following upper bound on $E_{P}(r)$ :

$$
E_{P}(r) \leq\left\{\begin{array}{l}
1, \quad r<D, \\
\exp \left[1-v_{1}(r)\right], \quad r \geq D
\end{array}\right.
$$

This upper bound is obtained by combining lower bound (53) on the cumulative coordination number $Z(r)$ and upper bound (128), and hence is a weaker upper bound on $E_{P}(r)$ than (128). As $d$ tends to infinity, we see that the upper bound (138) tends to the step function $\Theta(D-r)$, i.e.,

$$
E_{P}(r) \leq \Theta(D-r) \quad(d \rightarrow \infty) .
$$

Now we show that $E_{P}$ is bounded from below by the same step function in this limit, 
i.e.,

$$
E_{P}(r) \geq \Theta(D-r) \quad(d \rightarrow \infty) .
$$

To prove this, we note that the the lower bound (104) on $E_{P}(r)$ tends to a unit step function as $d$ becomes large [cf. Fig. 9] whose discontinuity location cannot exceed the zero of $1-Z(r), D_{0}$, which can be estimated to be given by

$$
D_{0}=\left[\frac{(d+2)^{2} \Gamma(1+d / 2)}{d K^{2}}\right]^{1 /(d+2)} .
$$

This estimate, which bounds the zero from below and becomes increasingly accurate as $d$ tends to infinity, is obtained by substituting the leading term of the asymptotic expansion (51) into $1-Z(r)$ and solving for $D_{0}$. For large $d, D_{0}$ has the asymptotic expansion

$$
D_{0}=\sqrt{\frac{d}{2 \pi e}}\left[1-\frac{\ln (d)}{2}+\mathcal{O}(1)\right] \quad(d \rightarrow \infty),
$$

Comparison of this expansion to that of the corresponding one for $D$ [cf. (55)] reveals that $D$ bounds $D_{0}$ from above for sufficiently large $d$ and $D_{0} \rightarrow D$ in the limit $d \rightarrow \infty$. Thus, the lower bound on $E_{P}(r)$ in this limit is given by (140). Combination of this lower bound with upper bound (139) leads to the following high-dimensional behavior:

$$
E_{P}(r) \rightarrow \Theta(D-r) \quad(d \rightarrow \infty),
$$

where we recall that $D$ grows like $\sqrt{d}$ for large $d$ [cf. (55)]. Following the analogous analysis using the lower bound (196) and upper bound (127) on $E_{V}(r)$, we can show

$$
E_{V}(r) \rightarrow \Theta(D-r) \quad(d \rightarrow \infty) .
$$

It is noteworthy that this analysis means that the lower bound (153) on the cumulative coordination number $Z(r)$ becomes exact in the limit $d \rightarrow \infty$. This in turn implies an "effective" pair correlation function $g_{2}^{*}(r)$ that tends to the following step function as $d$ tends to infinity:

$$
g_{2}^{*}(r) \rightarrow \Theta(r-D) \quad(d \rightarrow \infty) .
$$

This effective pair correlation function $g_{2}^{*}(r)$ is to be distinguished from the true pair correlation function (37), which tends to unity for distances beyond the length scale $D$, but remains a quadratic function of $r$ for small $r$. Because the effective pair correlation function is based on the behavior of $Z(r)$, which weights the true $g_{2}(r)$ by $r^{d-1}$ (due to the appearance of $s_{1}(r)$, the surface area of a sphere of radius $\left.r\right), g_{2}^{*}(r)$ tends to a step function in the high-dimensional limit. The fact that the oscillations of $g_{2}(r)$ seen in low dimensions (cf. Fig. 3) effectively vanish in the large- $d$ limit is consistent with the decorrelation principle [16], which, roughly speaking, states that unconstrained correlations that exist in low dimensions vanish as $d$ tends to infinity, and all higherorder correlation functions $g_{n}$ for $n \geq 3$ may be expressed in terms of $g_{2}$ within some small error.

We have already shown in (35) that the latter claim is true for any determinantal point process. The former claim is seen from the form of $g_{2}^{*}$ in (145), which immediately 
suggests that asymptotic unconstrained correlations in the Fermi sphere point process diminish with respect to increasing dimension $d$. In other words, $g_{2}$ flattens at unity for sufficiently large $r$ as $d$ becomes large, which implies that long-range correlations between any two particles in the system diminish with respect to increasing dimension, leaving only the small- $r$ correlations, which extend outward for a greater range in $r$ as $d$ increases. This conclusion in conjunction with (35) implies that for sufficiently large $d$ and for large particle separations, $g_{n} \approx \operatorname{det} \mathbb{I}=1$, where $\mathbb{I}$ is the $n \times n$ identity matrix. Therefore, all $n$-particle correlations also diminish for large particle separations and large $d$ in accordance with a decorrelation of the system. We remark that the fact that $g_{2}(r) \rightarrow 0$ as $r \rightarrow 0$ does not affect the statement of the decorrelation principle for the Fermi sphere point process; borrowing the language of quantum mechanics, these correlations arise from the constraint of antisymmetry in the many-particle wavefunction and therefore must be enforced in any dimension.

For sufficiently large $d$ this analysis implies that the system reduces to a sphere packing with an effective hard-core diameter equal to $D$. The connection to sphere packings implies that the fraction of space $\phi$ covered by the spheres at unit number density is bounded from above by the following inequality

$$
\phi \leq v_{1}(D / 2)=\frac{1}{2^{d}}
$$

Interestingly, Minkowski proved a lower bound on the coverage fraction of the densest lattice sphere packings that asymptotically is controlled by $1 / 2^{d}$ [43]. We remark on the significance of this result in Section 6.

5.4.3. Mean Nearest-Neighbor Distance We now obtain analytical estimates of the mean nearest-neighbor distance $\lambda$ at unit density using the general upper and lower bounds on $\lambda$ [cf. (111) and (112)]. Consider the arithmetic average of (111) and (112):

$$
\bar{\lambda}=\frac{\lambda_{L}+\lambda_{U}}{2} \text {. }
$$

For low dimensions, the arithmetic average of the upper and lower bounds for the Fermisphere point process provide reasonable estimates of $\lambda$, as seen in Table 1 for the first four space dimensions, which also includes the corresponding exact results. We see that the estimate $\bar{\lambda}$ captures the nonmonotone dependence of the mean nearest-neighbor distance with dimension. Moreover, the table shows that the upper bound becomes the dominant contribution to $\bar{\lambda}$ as $d$ increases and $\bar{\lambda}$ becomes increasingly accurate as the space dimensions increases.

Note use of the upper bound (138) enables us to obtain the following weaker but analytically solvable upper bound on $\lambda$ :

$$
\begin{aligned}
\lambda \leq \lambda_{U} \leq \lambda_{U *} & =D+\int_{D}^{\infty} \exp \left[1-v_{1}(r)\right] \mathrm{d} r \\
& =D\left[1+\frac{\Gamma(1 / d, 1) e}{d}\right]
\end{aligned}
$$


Point processes in arbitrary dimension

Table 1. Comparison of estimates of the mean-nearest neighbor distance $\lambda$ for the first four space dimensions of the Fermi-sphere point process at unit density to the corresponding "exact" values.

\begin{tabular}{cccccc}
\hline$d$ & Upper Bound & Lower Bound & Average of Bounds & Exact & $D$ \\
\hline 1 & 0.917808 & 0.658199 & 0.788003 & 0.725728 & 0.5 \\
2 & 0.688071 & 0.581193 & 0.634632 & 0.649823 & 0.564190 \\
3 & 0.670304 & 0.593975 & 0.632139 & 0.654511 & 0.620350 \\
4 & 0.687631 & 0.625009 & 0.656320 & 0.679561 & 0.670938 \\
\hline
\end{tabular}

where $\Gamma(x, a)$ is the incomplete gamma function. For large $d$, we have the asymptotic expression

$$
\begin{aligned}
\lambda_{U *} & =D\left[1+\frac{\Gamma(0,1) e}{d}+\mathcal{O}\left(\frac{1}{d^{2}}\right)\right] \\
& =D\left[1+\frac{0.5963473622 \ldots}{d}+\mathcal{O}\left(\frac{1}{d^{2}}\right)\right] \quad(d \rightarrow \infty) .
\end{aligned}
$$

Moreover, using the lower bound

$$
\lambda \geq \int_{0}^{D_{0}}[1-Z(r)] \mathrm{d} r
$$

where $D_{0}$ is the zero of $1-Z(r)$, and the aforementioned asymptotic analysis of the lower bound on $E_{P}(r)$, yields

$$
\lambda \geq D_{0} \quad(d \rightarrow \infty) .
$$

In summary, combination of the bounds (148) and (150) and the asymptotic expression (142), enables us to conclude that the mean nearest-neighbor distance approaches the length scale $D$ as $d$ becomes large, i.e.,

$$
\lambda \rightarrow D \quad(d \rightarrow \infty)
$$

which asymptotically grows like the square root of $d$ according to (55) and, as we concluded above, specifies the location of the step-function discontinuity of $E_{P}(r), E_{V}(r)$ and $g_{2}^{*}(r)$ in the large- $d$ limit. Table 1 shows that the length scale $D$ is already an accurate estimate of the mean nearest-neighbor distance for $d=4$.

It is noteworthy that the asymptotic mean nearest-neighbor-distance formula (152) is precisely the same as the asymptotic form of the mean nearest-neighbor distance of a Poisson point process. The latter for any dimension at unit density is given by $\Gamma(1+1 / d) \Gamma(1+d / 2)^{1 / d} / \sqrt{\pi}$ [37], which in the high-dimensional limit is exactly equal to $D$. The fact that the mean nearest-neighbor distance for the Fermi-sphere point process behaves like that of a Poisson point process in the high-dimensional limit is not surprising in light of the decorrelation principle [16].

Note that the expression for the mean nearest-neighbor distance $\lambda(\rho)$ for any density $\rho$ can be related to the corresponding quantity $\lambda(1)$ at unit density by the simple scaling relation

$$
\lambda(\rho)=\frac{\lambda(1)}{\rho^{1 / d}} .
$$


Figure 10 shows the mean nearest-neighbor distance as a function of density for various dimensions; the cases $d=1$ and $d=4$ are exact evaluations and the instance $d=17$ is obtained from the upper bound prediction (148) and the scaling relation (153), which is expected to be a highly accurate estimate.



Figure 10. The mean nearest-neighbor distance $\lambda$ as a function of density $\rho$ for various dimensions. The cases $d=1$ and $d=4$ are exact evaluations and the instance $d=17$ is obtained from the upper bound prediction (148) in conjunction with (153).

5.4.4. Large-r Behavior We conclude this section by making some remarks about the conditional nearest-neighbor functions $G_{V}(r)$ and $G_{P}(r)$. The fact that the exclusion probabilities $E_{V}(r)$ and $E_{P}(r)$ tend to the same step function in the high-dimensional limit implies that $G_{V}(r)$ and $G_{P}(r)$ have the same large- $r$ behavior as $d$ tends to infinity. In fact, our exact evaluations of $G_{V}(r)$ and $G_{P}(r)$ for a finite range of $r$ in low dimensions indicate that each function becomes linear in $r$ for large $r$ and the ratio $G_{P}(r) / G_{V}(r)$ tends to unity. Figure 11 shows our evaluations of both $G_{V}(r)$ and $G_{P}(r)$ for the first four space dimensions for the range $0 \leq r \leq 1.4$ (see our companion paper [14] for further numerical details).

In one dimension, one can show that the nearest-neighbor void functions are directly related to a radial function that is a solution to a second-order nonlinear differential equation [44. This differential equation can be evaluated exactly for small and large $r$. In particular, using this asymptotic analysis leads to the following large- $r$ behavior for $G_{V}(r)$ for the one-dimensional Fermi-sphere point process:

$$
G_{V}(r)=\frac{\pi^{2}}{2} r+\mathcal{O}\left(r^{-1}\right) \quad(r \rightarrow \infty)
$$

The coefficient $\pi^{2} / 2$ can be compared to the corresponding Wigner surmise prediction of $16 / \pi$, which is obtained via the one-dimensional relation that exactly links $E_{V}(r)$ to 

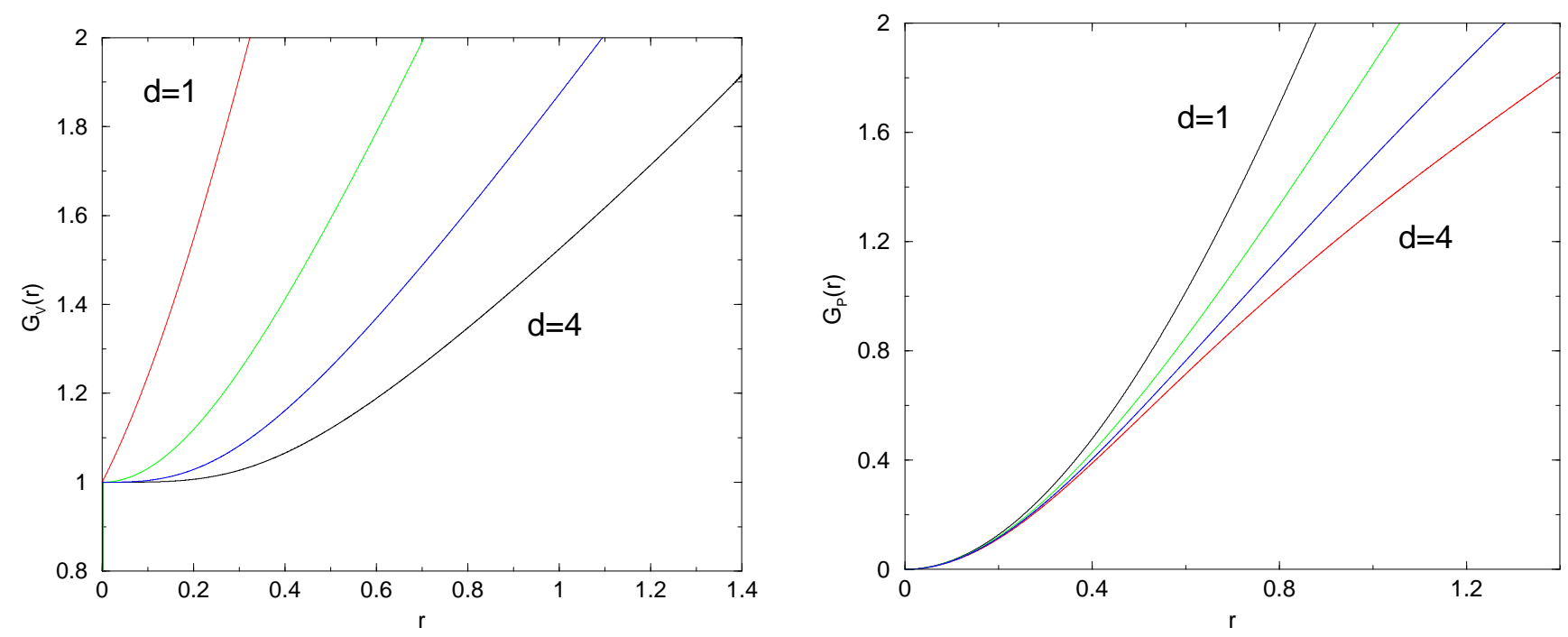

Figure 11. Exact calculations of both $G_{V}(r)$ and $G_{P}(r)$ for the first four space dimensions.

the gap distribution function $p(z)$ [45],

$$
E_{V}(r)=\int_{2 r}^{\infty}(z-2 r) p(z) \mathrm{d} z
$$

and Eq. (71) with $\beta=2$. Presumably, $G_{P}(r)$ has the same asymptotic behavior for $d=1$, a conclusion supported by our numerical results [14]. Interestingly, for both conditional probability functions to have the same linear behavior for large $r$ implies that the corresponding exclusion probability functions have the following large- $r$ behavior for finite $d$ :

$$
E_{V}(r)=E_{P}(r) \rightarrow \exp \left[-\kappa(d) r^{d+1}\right] \quad(r \rightarrow \infty),
$$

where $\kappa(d)$ is a positive $d$-dependent constant.

Thus, this analysis reveals that the probability of finding a large spherical cavity of radius $r$ for a Fermi-sphere point process in dimension $d$ behaves similar to that of a Poisson point process but in dimension $d+1$. For a Poisson point process, the constant $\kappa(d)=\pi^{(d+1) / 2} / \Gamma[(d+3) / 2]$, [cf. (90) and therefore if $E_{V}(r)$ for a Fermi-sphere point process in $\mathbb{R}^{d}$ for large $r$ behaved exactly like that for a Poisson point process in $\mathbb{R}^{d+1}$, then $G_{V}(r)$ would be given by

$$
G_{V}(r)=G_{P}(r)=\frac{\sqrt{\pi} \Gamma(d / 2)}{\Gamma[(d+1) / 2]} r+\mathcal{O}(1) \quad(r \rightarrow \infty) .
$$

For the first four space dimensions, the coefficients multiplying $r$ in (157) are given by $\pi=3.14 \ldots, 2, \pi / 2=1.57 \ldots$, and $4 / 3=1.33 \ldots$, respectively. This can be compared to the exact coefficients, which for $d=1,2,3$ and 4 are given by $\pi^{2} / 2=4.93 \ldots$, $2.815 \pm 0.048,1.597 \pm 0.043$, and $1.297 \pm 0.045$, respectively. The last three values are estimates that we have obtained based upon extrapolations from the evaluations of $G_{V}(r)$ (see Ref. [14] for further numerical details). We see that the probability of 
finding a large spherical cavity of radius $r$ in $\mathbb{R}^{d}$ is approximately the same as that for a Poisson point process in $\mathbb{R}^{d+1}$, implying linear growth of $G_{V}(r)$ or $G_{P}(r)$ for large $r$. It is not unreasoanble to conclude that this approximation becomes increasingly accurate as $d$ increases. Further justification for this remarkable behavior is given in Section 6 .

\section{Conclusions and Discussion}

We have obtained and characterized a new class of determinantal point processes in $\mathbb{R}^{d}$, the most general of which we call Fermi-shells point processes. The $n$-particle correlation functions for any $n$ and $d$, which completely characterize the point process, are determined analytically. We focused primarily on a special case, the Fermi-sphere point process, which in one dimension is identical to the point process that characterizes the spacings of the eigenvalues of the GUE (as well as the CUE), the conjectured spacings of the nontrivial zeros of the Riemann zeta function, and the positions of spin-polarized fermions in their ground state (i.e., completely filling the Fermi "sphere"). We are not aware of any correspondence of the general Fermi-shells point process in $\mathbb{R}^{d}$ for any $d \geq 2$ to random matrix theory or the zeros of any generalized zeta function in number theory. If our determinantal point processes have connections to random matrices in arbitrary space dimension, the latter must be non-Hermitian. For example, Ginibre [46] showed that there are two-dimensional determinantal point processes that correspond to complex eigenvalues of random non-Hermitian matrices. Thus, it is an open question whether the Fermi-shells point process has any correspondence to random matrices.

We analyzed in great detail properties of pair statistics, including the pair correlation function, the structure factor, and cumulative coordination number, as a function of spatial dimension $d$. The point processes for any $d$ are shown to be hyperuniform such that the structure factor (or power spectrum) $S(k)$ has a nonanalytic behavior at the origin given by $S(k) \sim|k|(k \rightarrow 0)$. The latter result implies that the pair correlation $g_{2}(r)$ tends to unity for large pair distances with a decay rate that is controlled by the power law $1 / r^{d+1}$. In three dimensions, such a dominant power-law decay of $g_{2}(r)$ is a well-known property of bosonic systems in their ground states [10, 12 ] and, more recently, has been shown to characterize maximally random jammed sphere packings [13. We also graphically displayed one- and two-dimensional realizations of the point processes in order to vividly reveal their "repulsive" nature and demonstrated that they can be characterized by an effective "hard-core" diameter that grows like the square root of $d$.

Our study of the nearest-neighbor functions of the Fermi-sphere point process resulted in some noteworthy conclusions. For example, we have seen that the probability of finding a large spherical cavity of radius $r$ in $\mathbb{R}^{d}$ is approximately the same as that for a Poisson point process in $\mathbb{R}^{d+1}$, implying linear growth of $G_{V}(r)$ or $G_{P}(r)$ for large $r$. This is a remarkable result because it represents the first class of nontrivial point processes that we are aware of whose conditional nearest-neighbor functions do not asymptote to a constant; see Refs. [38, 47, 48] and [45] for examples of correlated equilibrium and 
nonequilibrium point processes that possess $G_{V}(r)$ or $G_{P}(r)$ with constant asymptotes, respectively. It is clear that the unusual property of linear growth of $G_{V}$ or $G_{P}$ for large $r$ is due to the long-range nature of the repulsive interactions. Since a Fermi-sphere point process in $\mathbb{R}^{d}$ is always hyperuniform, i.e., large wavelength density fluctuations vanish, the probability of finding a large spherical cavity must be smaller than the corresponding quantity for any point process that is not hyperuniform, such as a Poisson point process in $\mathbb{R}^{d}$. The probability of finding a large spherical cavity is of course smaller in a Poisson point process in dimension $d+1$ compared to that in dimension $d$. Moreover, it is easy to show that the asymptotic form (156) for $E_{V}(r)$ or $E_{P}(r)$ for large $r$ is always between the aforementioned corresponding rigorous upper and lower bounds on the exclusion probabilities.

We also found that the Fermi-sphere point process becomes a sphere packing in the high-dimensional limit with an effective hard-core diameter equal to the length scale $D$ [cf. (54)]. Thus, the fraction of space $\phi$ covered by the spheres at unit number density is bounded from above by $1 / 2^{d}$. This coverage fraction has a special significance in the study of sphere packings; it arises not only in Minkowski's famous century-old lower bound on the density of the densest lattice sphere packings [43] but in lower bounds for saturated and disordered packings [16] as well as the highest achievable density in the "ghost" random sequential addition packing [8]. It should be noted, however, that there is strong evidence that there exist disordered sphere packings with $\phi$ not only greater than $1 / 2^{d}[49$, 51, 52, 50] but with densities that exponentially improve on Minkowski's lower bound [16, 23].

Elsewhere [14] we report results on the extremes of the nearest-neighbor statistics as well as Voronoi statistics of the Fermi-sphere point processes in the first four space dimensions. In other work, we will quantify clustering and percolation properties of Fermi-sphere point processes.

\section{Acknowledgments}

The authors are grateful to Peter Sarnak and Juan Maldacena for useful discussions. This work was supported by the National Science Foundation under Grant No. DMS0804431.

\section{Appendix A. On the Presence of Intrinsic $n$-Particle Interactions (with $n \geq 3$ ) for General Determinantal Point Processes}

Our purpose here is to show that the $n$-particle probability density function for an arbitrary determinantal point process, even in one dimension, cannot be written as a Boltzmann factor of $N$ classical particles interacting through one- and two-body potentials at a finite temperature. Although this claim is true for each of the canonical ensembles of random-matrix theory, we show via a counterexample in one dimension that intrinsic $n$-particle interactions with $n \geq 3$ are generally necessary to describe the 
system.

It is relatively straightforward to express the $n$-particle probability density function for the CUE as a Boltzmann factor of a classical system of pairwise-interacting particles. In analogy with the formalism of the one-dimensional Fermi-sphere point process introduced in Section 4.1, we fill the Fermi line at a constant density. Thus, may write [3]:

$$
\operatorname{det}\left[\exp \left(\mathrm{i} n x_{m}\right)\right]_{n, m}=\prod_{n<m}\left[\exp \left(\mathrm{i} x_{n}\right)-\exp \left(\mathrm{i} x_{m}\right)\right]
$$

therefore,

$$
\begin{aligned}
\left|\operatorname{det}\left[\exp \left(\mathrm{i} n x_{m}\right)\right]_{n, m}\right|^{2} & =\exp \left[-2 \sum_{n<m} \ln \left|\exp \left(\mathrm{i} x_{n}\right)-\exp \left(\mathrm{i} x_{m}\right)\right|\right] \\
& =\exp \left[-2 \sum_{n<m} \ln \left|\sin \left(x_{n}-x_{m}\right) / 2\right|\right] .
\end{aligned}
$$

For small eigenvalue separations $\Delta x_{n m}=x_{n}-x_{m}$, the result in (A.3) reduces to the form:

$$
\left|\operatorname{det}\left[\exp \left(\mathrm{in} x_{m}\right)\right]_{n, m}\right|^{2} \approx \exp \left[-2 \sum_{n<m} \ln \left|\left(\Delta x_{n m} / 2\right)\right|\right],
$$

and the probability distribution of the CUE eigenvalues can indeed be written as a pairwise interacting potential, which in this example is logarithmic for small particle separations. It is important to note that this reformulation of the probability density is very peculiar to the method of filling the Fermi sphere. Suppose instead that we decide to fill only the states $n=0,2,3, \ldots$; i.e., the state $n=1$ is skipped. We may then express the three-body form of the probability density as:

$$
\begin{gathered}
\left|\operatorname{det}\left[\exp \left(\operatorname{in} x_{m}\right)\right]_{n, m}\right|^{2}=\frac{64}{(2 \pi)^{3}}[3+2 \cos (x-y)+2 \cos (x-z)+2 \cos (y-z)] \\
\times \sin \left(\frac{x-y}{2}\right)^{2} \sin \left(\frac{x-z}{2}\right)^{2} \sin \left(\frac{y-z}{2}\right)^{2},
\end{gathered}
$$

where we have used $x, y$, and $z$ to represent $x_{0}, x_{2}$, and $x_{3}$; the subscripts denote the state $n$ of each particle. The last three factors have the form of the pair interaction in (A.3). To check whether the pre-factor containing cosines can be written in the same form, we write:

$$
V(x, y, z)=-\ln [3+2 \cos (x-y)+2 \cos (x-z)+2 \cos (y-z)] .
$$

Assume that $V(x, y, z)=v(x, y)+v(y, z)+v(z, x)$. It must then be true that:

$$
\begin{aligned}
v(x, y) & =\frac{1}{2}\left[V(x, y, y)-\frac{1}{3} V(y, y, y)\right] \\
& =\frac{\ln (3)}{3}+\frac{1}{2} \ln [5+4 \cos (x-y)],
\end{aligned}
$$

but by substituting this expression into (A.6), we see that we do not recover the original functional form. Therefore, we have shown by contradiction that for general 
determinantal point processes, the interaction potential must contain at least intrinsic three-body terms.

\section{References}

[1] Dyson F J, Statistical Theory of the Energy Levels of Complex Systems. I, 1962 J. Math. Phys. 3 140

[2] Dyson F J, Correlations between Eigenvalues of a Random Matrix, 1970 Comm. Math. Phys. 19 235

[3] Mehta M L, 1967 Random Matrices (New York: Academic Press)

[4] Montgomery H L, The pair correlation of zeros of the zeta function, in: 1973 Proc. Sympos. Pure Math. vol. 24 (Providence: AMS) pp. 181-193

[5] Odlyzko A M, On the Distribution of Spacings Between Zeros of the Zeta Functions, 1987 Math. Comput. 48 273; Odlyzko A M, The $10^{22}$-nd zero of the Riemann zeta function, in: van Frankenhuysen M and Lapidus M L, 2001 Dynamical, Spectral, and Arithmetic Zeta Functions (Providence: AMS) pp. 139-144

[6] Rudnick Z and Sarnak P, Zeros of principal L-functions and random matrix theory, 1996 Duke Math. J. 81269

[7] Katz N M and Sarnak P, Zeros of zeta functions and symmetry, 1999 Bull. Amer. Math. Soc. 36 1

[8] Torquato S and Stillinger F H, Exactly solvable disordered sphere-packing model in arbitrarydimensional Euclidean spaces, 2006 Phys. Rev. E $\mathbf{7 3} 031106$

[9] Feynman R P, 1998 Statistical Mechanics (Boulder, CO: Westview Press)

[10] Feynman R P, Atomic Theory of the Two-Fluid Model of Liquid Helium, 1954 Phys. Rev. 94262

[11] Feynman R P and Cohen M, Energy Spectrum of the Excitations in Liquid Helium, 1956 Phys. Rev. 1021189

[12] Reatto L and Chester G V, Phonons and the Properties of a Bose System, 1967 Phys. Rev. 155 88

[13] Donev A, Stillinger F H and Torquato S, Unexpected Density Fluctuations in Jammed Disordered Sphere Packings, 2005 Phys. Rev. Lett. 95090604

[14] Scardicchio A, Zachary C E and Torquato S, 2008 submitted for publication

[15] Stoyan D, Kendall W S and Mecke J, 1995 Stochastic Geometry and Its Applications (New York: Wiley)

[16] Torquato S and Stillinger F H, New Conjectural Lower Bounds on the Optimal Density of Sphere Packings, 2006 Exp. Math. 15307

[17] Beck J, Irregularties of distribution I, 1987 Acta Mathemtica 1591

[18] Torquato S and Stillinger F H, Local density fluctuations, hyperuniformity, and order metrics, 2003 Phys. Rev. E 68041113

[19] Gabrielli A, Jancovici B, Joyce M, Lebowitz J L, Pietronero L and Labini F S, Generation of primordial cosmological perturbations from statistical mechanical models, 2003 Phys. Rev. D 67 043506

[20] Gabrielli A and Torquato S, Voronoi and void statistics for superhomogeneous point processes, 2004 Phys. Rev. E $\mathbf{7 0} 041105$

[21] Gabrielli A, Joyce M and Torquato S, Tilings of space and superhomogeneous point processes, 2008 Phys. Rev. E $\mathbf{7 7} 031125$

[22] Torquato S and Stillinger F H, Controlling the Short-Range Order and Packing Densities of ManyParticle Systems, 2002 J. Phys. Chem. B 106 8354; ibid, 200210611406

[23] Scardicchio A, Stillinger F H and Torquato S, Estimates of the optimal density of sphere packings in high dimensions, 2008 J. Math. Phys. 49043301

[24] A sphere packing is a point process in which there is a minimal positive distance between any pair of points. According to Ref. [16, a disordered sphere packing in $\mathbb{R}^{d}$ is one in which the pair 
correlation function $g_{2}(\mathbf{r})$ decays to its long-range value of unity faster than $|\mathbf{r}|^{-d-\varepsilon}$ for some $\varepsilon>0$.

[25] Sarnak P and Strombergsson A, Minima of Epstein's Zeta function and heights of flat tori, 2006 Inventiones Math. 165115

[26] Torquato S and Stillinger F H, New Duality Relations for Classical Ground States, 2008 Phys. Rev. Lett. 100020602

[27] Macchi O, The Coincidence Approach to Stochastic Point Processes, 1975 Adv. Appl. Probab. 783

[28] Soshnikov A, Determinantal random point fields, 2000 Russian Mathematical Surveys 55923

[29] Hough J B, Krishnapur M, Peres Y and Virág B, Determinantal Processes and Independence, 2006 Probab. Surveys $\mathbf{3} 206$

[30] Johansson K, Determinantal Processes with Number Variance Saturation, 2004 Comm. Math. Phys. 252111

[31] Burton R and Permantle R, Local Characteristics, Entropy and Limit Theorems for Spanning Trees and Domino Tilings Via Transfer-Impedances, 1993 Ann. Probab. 211329

[32] Horn R A and Johnson C R, 2005 Matrix Analysis (Cambridge: Cambridge UP)

[33] Note that for arbitrary density $\rho$, the corresponding pair correaltion function is still given by (43) but with $K=\rho^{1 / d} 2 \sqrt{\pi} \Gamma(1+d / 2)^{1 / d}$, which reduces to (40) for unit density.

[34] Gioev D and Klich I, Entanglement Entropy of Fermions in Any Dimension and the Widom Conjecture, 2006 Phys. Rev. Lett. 96100503

[35] Costin O and Lebowitz J L, On the Construction of Particle Distributions with Specified Single and Pair Densities, 2004 J. Phys. Chem. B. 10819614

[36] Torquato S and Lu B, Chord-length distribution function for two-phase random media, 1993 Phys. Rev. E 472950

[37] Torquato S, 2002 Random Heterogeneous Materials: Microstructure and Macroscopic Properties (New York: Springer-Verlag)

[38] Torquato S, Lu B and Rubinstein J, Nearest-neighbor distribution functions in many-body systems, 1990 Phys. Rev. A 412059

[39] McWeeny R, Some Recent Advances in Density Matrix Theory, 1960 Rev. Mod. Phys. 32335

[40] Slater J C, A Simplification of the Hartree-Fock Method, 1951 Phys. Rev. 81385

[41] Boyd R J and Coulson C A, The Fermi hole in atoms, 1974 J. Phys. B: Atom. Molec. Phys. 7 1805

[42] Schwabl F, 2005 Advanced Quantum Mechanics 3rd ed. (Berlin: Springer-Verlag)

[43] Minkowski H, Diskontinuitätsbereich für arithmetische Äquivalenz, 1905 J. reine angew. Math. 129 220

[44] Tracy C and Widom H, Level-Spacing Distributions and the Airy Kernel, 1994 Commun. Math. Phys. 159151

[45] Rintoul M D, Torquato S and Tarjus G, Nearest-neighbor statistics in a one-dimensional random sequential adsorption process, 1996 Phys. Rev. E $\mathbf{5 3} 450$

[46] Ginibre J, Statistical Ensembles of Complex, Quaternion, and Real Matrices, 1965 J. Math. Phys. 6440

[47] Torquato S and Lee S B, Computer simulations of nearest-neighbor distribution functions and related quantities for hard-sphere systems, 1990 Physica A 164347

[48] Lu B and Torquato S, Nearest-surface distribution functions for polydispersed particle systems, 1992 Phys. Rev. A 455530

[49] Parisi G and Zamponi F, Amorphous packings of hard spheres for large space dimension, $2006 \mathrm{~J}$. Stat. Mech.: Theory Exp. P03017

[50] Zamponi F, Some recent theoretical results on amorphous packings of hard spheres, 2007 Phil. Mag. 87485

[51] Skoge M, Donev A, Stillinger F H and Torquato S, Packing hyperspheres in high-dimensional Euclidean spaces, 2006 Phys. Rev. E $\mathbf{7 4} 041127$

[52] Torquato S, Uche O U and Stillinger F H, Random sequential addition of hard spheres in high 
Euclidean dimensions, 2006 Phys. Rev. E 74061308 